\title{
A Real-Time Early Warning Seismic Event Detection Algorithm Using Smart Geo-Spatial Bi-Axial Inclinometer Nodes for Industry 4.0 Applications
}

\author{
Hasan Tariq ${ }^{1, *}$, Farid Touati ${ }^{1}$, Mohammed Abdulla E. Al-Hitmi ${ }^{1}$, Damiano Crescini ${ }^{2}$ and \\ Adel Ben Mnaouer ${ }^{3}$ (D) \\ 1 Department of Electrical Engineering, College of Engineering, Qatar University, 2713 Doha, Qatar \\ 2 Dipartimento di Ingegneria delI'Informazione, Brescia University, 25121 Brescia, Italy \\ 3 Department of Computer Engineering and Computational Sciences, Faculty of Engineering, Applied \\ Sciences and Technology, Canadian University Dubai, 117781 Dubai, UAE \\ * Correspondence: hasan.tariq@qu.edu.qa; Tel.: +974-50419852
}

Received: 3 August 2019; Accepted: 29 August 2019; Published: 4 September 2019

\begin{abstract}
Earthquakes are one of the major natural calamities as well as a prime subject of interest for seismologists, state agencies, and ground motion instrumentation scientists. The real-time data analysis of multi-sensor instrumentation is a valuable knowledge repository for real-time early warning and trustworthy seismic events detection. In this work, an early warning in the first 1 micro-second and seismic wave detection in the first 1.7 milliseconds after event initialization is proposed using a seismic wave event detection algorithm (SWEDA). The SWEDA with nine low-computation-cost operations is being proposed for smart geospatial bi-axial inclinometer nodes (SGBINs) also utilized in structural health monitoring systems. SWEDA detects four types of seismic waves, i.e., primary (P) or compression, secondary (S) or shear, Love (L), and Rayleigh (R) waves using time and frequency domain parameters mapped on a 2D mapping interpretation scheme. The SWEDA proved automated heterogeneous surface adaptability, multi-clustered sensing, ubiquitous monitoring with dynamic Savitzky-Golay filtering and detection using nine optimized sequential and structured event characterization techniques. Furthermore, situation-conscious (context-aware) and automated computation of short-time average over long-time average (STA/LTA) triggering parameters by peak-detection and run-time scaling arrays with manual computation support were achieved.
\end{abstract}

Keywords: applied methods; earthquake; seismic waves; real-time detection; early warning; inclinometers; Internet of Things (IoT)

\section{Introduction}

Natural disasters occur on the globe every year with earthquakes and floods being the most devastating and horrible on the loss and damage benchmarks. The number of people reported affected by natural disasters (564.4 million) was the highest since 2006, as compared to the last 10 years [1], amounting to 1.5 times its annual average (224 million). The estimates of natural disaster economic damages (US\$154 billion) place last year as the fifth costliest since 2006, 12\% above the 2006-2015 annual average registered in the CRED database. Earthquakes or seismic events have proven to be the most obvious and recurring in all [2] the natural disasters, i.e., 14,568 in 2018. The top of the chart was in Indonesia on 28 September 2018, with 2256 death tolls. Real-time early warning alarms and intimation would have reduced these life and financial loss numbers. The event characterization and detection would have resulted in countermeasures against these disasters and considerable safety would have been observed. The necessity of an expeditious mechanism was observed in $[1,2]$ that 
could gradually create awareness using amplitude thresholding and frequency filtering in a swift manner in the form of early-stage warnings for human life and asset safety.

Recent advancements [3] in the earth, planetary, and space studies have created new horizons for real-time early warning studies of disasters. The subsurface [4] camera (SAMERA) capitalizing the three-layered distributed iterative computing paradigm for three prototypes, (1) travel-time seismic tomography (TomoTT); (2) migration-based microseismic imaging (MMI); (3) ambient noise seismic imaging (ANSI), was very comprehensive in 3D velocity maps and subsurface realization with a need for early warning and orientation compensation. The development of an integrated earthquake simulation system for Istanbul and utilization of ambient vibration measurements for risk assessment [5] at an urban scale for Beirut had created a need for a geospatially oriented seismic early warning awareness. The four degrees of freedom [6] ground motion simulation platform (GMSP) was also a comprehensive contribution to a gap of real-time ground motion detection capabilities. The design challenges with the Marmara Sea case study [7] for such a system that was resourceful in major seismic events like volcanic, lithospheric, and tsunamic activity could only be provisioned in ubiquitous scenarios, i.e., geo-spatially present in urban infrastructure.

Recent achievements [8] in geo-seismic or ground motion instrumentation and multi-variable urban-scale telemetry created the potential for damage post-analysis by immense computation at the national level with high costs. The GPS assisted geo-magnetic storm technique [9] and broadband seismic network health monitoring method needed improvement focused on the real-time systems-level approach. A few seconds to less than a minute warning before the arrival of damaging ground motion needed minimum calculations and discrete mechanism defined in [10] as multi-objective SHM prediction algorithm (MOSPA) needed further enrichment for geo-seismic cases that have not been observed in the literature before. Real-time catastrophic incidents in densely populated, commercial, and industrial zones particularly needed local [11] early warning systems, particularly for mitigating an earthquake that is aligned with strategic facilities [12] and lifelines of buildings in form of structural health monitoring (SHM) and indigenous structural conditions sensing (ISCS) nodes. The desideratum of low noise, high resolution, and a sampling rate ubiquitous unit solution capable of long-range error compensating communication was observed in [4-12], accommodating for early warning [1-3] and orientation error compensation.

ISCS aimed to provide prognosis [13] in a smart way to make a non-destructive evaluation (NDE), precise long-haul monitoring of angular displacements, and critical frequencies. Geo-seismic event detection falls in the category of NDE [14] that follows four basic strategies: time series, a condition triggered, load effect, and sensors orientation schemes. Almost all the physical variables [15] are keystones in the NDE of structures, i.e., temperature, pressure, humidity, displacement, and vibration using cameras, Bragg grating fiber optic sensors, electrochemical(corrosion), laser Doppler vibrometer (LVD), accelerometers [16], strain gauges, linear variable differential transformers (LVDT), piezo-electric load cells, and thermocouples. A simple, low cost, diligent, and robust high resolution $\pm 0.0000 \mathrm{X}$ angular displacement monitoring solution with 12 24-bit configurable analog to digital converter (ADC) was needed to accommodate [1-13] in a plethora of NDE sensors [14-16] only for fundamental geo-mechanics early warnings and least computation assessments.

Recent review work by [17] concluded that the five generic approaches to NDE are (1) wavelet transform approach, (2) empirical mode decomposition (EMD) approach, (3) Hilbert-Huang transform (HHT) approach, (4) parametric system identification (PSI) approach, (5) the peak picking approach, and (6) damage identification. Detection of abrupt changes [18] in structural integrity using peak picking methods was more hardware implementable [19] for pre-evaluation or NDE. The semi-automatic peak picking technique SegNet [20] based on convolutional neural networks (CNNs) was successful in peak picking. SegNet needed training of ANNs for image processing resulting in high computation cost that limited its applicability for real-time detection applications with hardware calibration. The machine learning-based earthquake early warning (EEW) approach [21] based on generative adversarial network (GAN) critic, as an automatic feature extractor-based discriminator, could recognize $99.2 \%$ of the 
earthquake $\mathrm{P}$ waves and $98.4 \%$ of the noise signals. The EEW had extreme pre-processing computation costs and real-time detection limitations on physically embedded systems. A very valuable work [22], illustrating the weighted genetic feature search algorithm, had gaps in the real-time application of the five techniques using wired and wireless schemes. The work [23] with (1) vibration-based method, (2) impedance-based method, (3) data fusion method, and (4) inverse methods used in wired techniques and smart sensing technology comprised of multi-sensor motes utilized in wireless techniques and served an excellent ISCS directive. The crave for time series-based least-cost applied computation was needed for ISCS or any geo-mechanics sensing unit due to the lapse in real-time applicability [17-23] of complex methods.

In the light of the study carried out in this work from ISCS for [1-13] requirements, strategies, methods, and techniques, the importance of positioning and orientation [24] of data acquisition points was the vital factor for implementing any algorithm for detailed feature extraction and study. The reliability equations in [25] were a noticeable contribution for ISCS and should be evaluated by R composed of (1) intrinsic capability (IC), (2) application parameters (AP), and (3) human factor (HF) governed by probability of false alarm (POFA), and (4) probability of detection (POD). Further study of SHM variables [26] led to the fact that vibration and acoustic factors by are most vital and instantaneous that can demolish grids of structures in the nick of time, all the rest are transients, i.e., only contribute to aging and decay. The review of eight [27] methods-(1) vibration-based damage identification (VBDI) methods; (2) natural frequency-based methods (NFM), (3) mode shape-based methods (MSM), (4) mode shape curvature/strain mode shape-based methods (MSCS), (5) dynamically measured flexibility-based methods (DMFM), (6) matrix update based methods (MUM), (7) non-linear methods (NLM), and (8) artificial neural network methods (ANNM)—applied to ISCS can be very useful in interpreting the global displacement response of regional and urban structures to identify local changes in it. A common gap observed in [24-27] was an adaptive event capturing pre-triggering and post-triggering that guided the entire data manipulation leading to immeasurable real-time delays and computational costs.

On the other hand, the real-time event capturing holds a momentous significance in the credibility of any real-world event detection application. The standard short-time average and long-time average (STA/LTA) triggering algorithm [28] has been the one most applied and used by state agencies [29] in the USA and EU. STA/LTA is a very resource-efficient algorithm. The adaptive STA/LTA used by the waveform correlation event detection [30] system and the Hoadley gas field, Alberta, Canada [31], was the nearest contestant with much more resource requirement. The S/L-Kurt method [32] was more effective than the standard (STA/LTA) mechanism for arrivals because it reduces any bias in the short-term kurtosis (STK) and long-term kurtosis (LTK) window limited to desktop machines in Industry 4.0 applications. The S/L-Kurt-assisted real-time seismic evaluation [33] and interpretation [34] is considered as, so far, the most critical of all the displacement-based SHM procedures using time series analysis. The nature of displacement data is that it consists of time series and is further modularized into a pattern of windows by application of the Dempster-Shafer theory of evidence [35], handled through the matrix and statistical techniques. The demonstration and significance [36] of window sizes for STA and MER; STK for MCM and LTA; and LTK and PAI-K for field microseismic data sets streamlined by IKK's method, ZTR's method, JER-AIC, Akazawa's method, and S/L-Kurt [37] were found to be more precise for constraint cases with a gap in the networked sensor detection mechanisms. However, the contribution [38] was based on a machine with i7-4720HQ CPU having clock $2.60 \mathrm{GHz}$ and 16 GB RAM size that limited its canvas in ubiquitous Industry 4.0 applications due to complex and high computation costs as well as automated ADC calibration. The geo-seismic sensing capabilities for [1-13] and real-time limitations in [13-38] methods had sequenced output gaps based on [1-3] enormities. The clustered and networked ISCS assessments raised new complexities in assessments only possible for geographical area network solutions at the cloud level [9].

The application constraint methodological sequence [17-38] was very important in seismic event characterization as an earthquake after following and considering all the above discussed methodologies 
and procedures. The application-specific [33] seismic sensor nodes and body area network (BAN) makes SHM very intelligent and robust towards seismic event detection and identification and makes the choice of algorithms easier at the cost of flexibility in the SHM system. Recent works [39] realized the utility of (a) adaptive iterative hard thresholding (AIHT), (b) support vector machines (SVM), and (c) the consensus and innovations [40] sequential probability ratio test (CISPRT) [37] and proposed four different algorithms, namely: (1) the least-favorable-density CISPRT; (2) the median CISPRT; (3) the M-CISPRT; and (4) the myriad CISPRT. The approaches [36-38] were very plausible for uniformly distributed repetitive series of sensors data but required real-time testing on hardware through the instrumentation of micro-displacements. The Green's functions [38] played a vital role in surface wave tomography of the Western United States from ambient seismic noise for Rayleigh and Love wave phase velocity maps. The assistance of Green's functions in ADC parameter optimization [41] could lead to better results in Industry 4.0-based seismic detection solutions for desktop-scale machines.

A promptly swift warning and geo-seismic waves detection solution was needed to address the following challenges:

(a) A geo-seismic constraint sensing node with ADC comprised of 2+ times higher sampling frequency for seismic signals as per the Nyquist criterion and a resolution of $\pm 0.0000 \mathrm{X}$.

(b) Normalization of a single geo-seismic sensing node (GSSN) or ISCS magnitude offsets induced by placement errors.

(c) Normalization of clustered GSSNs or ISCS magnitudes complex orientation errors.

(d) Heterogeneous surface orientation errors were reflected in entire measurements and geo-seismic data processing.

(e) Gaussian or pseudo-random noise issues in the form of high frequencies and nano- or micro-seismic angular displacement anomalies.

(f) Sequential detection of incremental displacement amplitudes to reduce post-computation costs and predict the upcoming threats [11] as primary, secondary, and tertiary alarms.

(g) Adaptive amplitude real-time thresholds mapping to reduce incremental filtering costs.

(h) Real-time estimation of pre-triggering and post-triggering parameters for recording or event capturing algorithms based on amplitudes, number of samples, and obliged frequencies.

(i) Runtime or real-time similarity in amplitudes and frequencies with existing time series of earthquakes that happened in the past in parallel and compare triggering time intervals as discrete occurrences instead.

(j) The geometrical exploration of seismic wave kinematics (frequency and amplitudes of two-dimensional angular displacements) for maximum details required for the geo-seismic realization using algorithmic calibration.

(k) Methodological, adaptive, and event-driven sensor calibration and measurement optimization capability nodes are enablement towards Industry 4.0 applications.

(1) Real-time remote calibration of ADC parameters to optimize low-cost geo-seismic detection using error-compensated, de facto, de jure industry-standard communication buses. This capability constitutes a steppingstone towards Industry 4.0-based condition monitoring benchmarks.

(m) Sustainable distant and chronological optimization of real-time seismic event detection.

(n) Adaptive real-time scaling of sensors to zoom+/zoom- in case of micro/macro magnitudes for iterative optimization.

(o) The real-time sensor calibration and recording triggering feature for unit sensor nodes as well as sensor clusters is the Industry 4.0 application benchmark for SWEDA.

The solution proposed in this work, featuring the above (b)-(o) enhancements, constitutes a sequential synergic combination of methods to address the above real-time challenges called real-time seismic wave event detection algorithm (SWEDA). The implementation of SWEDA was realized by developing a geo-seismic novel node with novelties (a) and (j) to(n). This work is organized as: 
1. Real-time seismic wave event detection algorithm (SWEDA) for cyber-physical systems.

2. Smart geospatial bi-axial inclinometer nodes (SGBINs) application design for SWEDA.

In Section 2, the methodological process of SWEDA is explained. Section 3 is dedicated to the SGBIN application design to achieve credible results from SWEDA. Section 4 is based on a case study presented with a practical implementation of SWEDA using SGBINs at the campus level, which demonstrated the capabilities of SWEDA and SGBIN clusters at Qatar University, Doha.

\section{Methodology Architecture}

Let us consider a time series vector $\mathrm{N}(\mathrm{t})$ as the sample space of $\mathrm{t}$ variables, such that $\mathrm{t}$ is not null, belongs to the set of real numbers, and is acquired from sensors as time series. The first step is to train patterns of seismic waves that can be compared with $\mathrm{N}(\mathrm{t})$ in real-time to characterize the seismic event. Earthquake, the most important special case in refractive seismology, be $\mathrm{E}(\mathrm{t})$ and is given by the characteristic piecewise random function:

$$
\mathrm{E}(\mathrm{t})=\left\{\begin{array}{l|l}
W 1(t 1) & 1 s<t 1<17 s \\
W 2(t 2) & 4.5 s<t 2<50 s \\
W 3(t 3) & 23 s<t 3<100 s
\end{array}\right\}
$$

In Equation (1), W1, W2, and W3 are the three seismic wave types that are time-stamped. $W 1$ is the p-wave, $W 2$ is the $\mathrm{S}$-wave, and $W 3$ is the surface wave. $\mathrm{t} 1, \mathrm{t} 2$, and $\mathrm{t} 3$ are the arrival times of $W 1$, $W 2$, and $W 3$ respectively.

A standard or characteristic earthquake seismograph pattern is exhibited in Figure 1. First, P-waves arrive, second, S-waves, and then surface waves (Rayleigh (R) or Love (L) waves). The sequential procedure for early warning and detection of an earthquake needs the realization of sequential events in the fashion of occurrence called SWEDA. The steps of SWEDA are given as:

1. Gradient map auto-calibration (GMAC)

2. Micro-seism or seismic noise filtration (SNF)

3. Peak detection sequence (PDS)

4. Autoregressive pattern mapping sequence (APMS)

5. Scaling coefficients array generation sequence (SCAGS)

6. STA/LTA arrays windows sequence (SLAWS)

7. Earthquake probabilistic sequence (EPS)

8. Seismic features extraction (SFE)

9. ADC scaling/range and communication bus configuration

SWEDA was programmed and tested on three-layered devices for constrained application testing:

1. MATLAB for Desktop PC Workstation.

2. MATLAB Coder for Raspberry Pi.

3. Python 2.7 for Single Board Computer (Raspberry Pi).

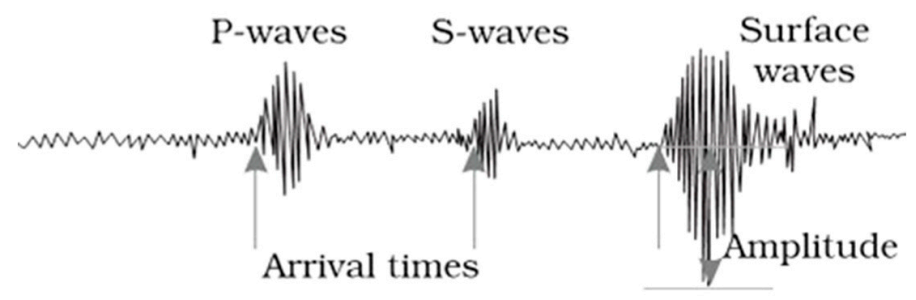

Figure 1. Earthquake seismograph and wave arrivals [3]. 
The sequence of operations was as characteristic as the seismic wave patterns or geo-seismic faults that will be characterized and detected by the algorithm. First, the placement map of sensors was created for assigning all current positions equal to zero so that whatever the type of plain and surface is the initial state vector in the time domain as time series. The noise filtering, thresholding, peak-detection, event capturing, or recording algorithm triggering, as well as auto-scaling for events using computed windows and seismic event extraction, was automated. In the case of high-resolution needs and high data rates, the algorithm re-configures itself at $t_{1}>t_{0}$, i.e., $t_{0}$ is the time at which the first sample set was processed in SWEDA and $t_{1}$ is the time at which the second sample set was processed by SWEDA. The entire process is summarized in Figure 2.

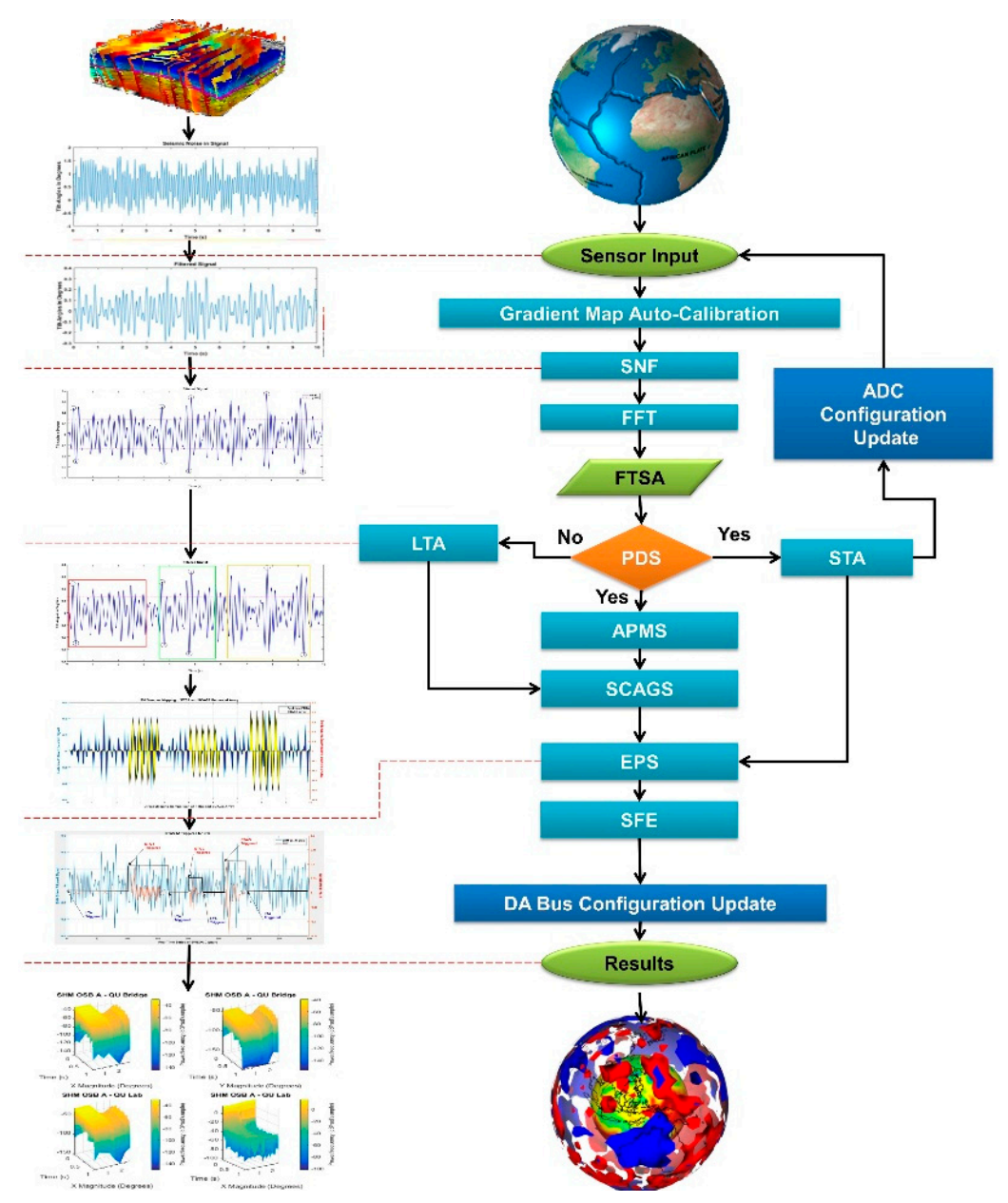

Figure 2. Seismic wave event detection algorithm (SWEDA) process flow diagram.

\subsection{Gradient Mapping and Auto-Calibration (GMAC)}

It was impossible in $100 \%$ sensor grid scenarios to achieve zero magnitudes, as the crust of the earth is like a curvature and structures placed on earth are mostly at a non-uniform distance from the center of the earth. For orientation, SWEDA takes the value of sensor variables as zero by subtracting the existing magnitudes. Let the $\mathrm{n}$ sensor variables at the time of placement have a vector $\mathrm{S}$ with a magnitude at $t=0$ written as $|\mathrm{S} 0|$. Let SP1 be the processed value at the start of SWEDA which is given by

$$
\mathrm{SP} 1=|\mathrm{S} 1|-|\mathrm{S} 0|=0 .
$$

In Equation (2), SP1 will always be zero and the sensor placement vector will be stored in the memory for reference. It will relieve the extra data processing operations like enveloping, normalization, magnitude averaging, variance, etc. 
Figure 3 clarifies the gradient map auto-calibration (GMAC) operation on SGBIN clusters to achieve zero-offsets by subtracting all the gradients from all the sensor values in the time series, which reduced computation complexities.

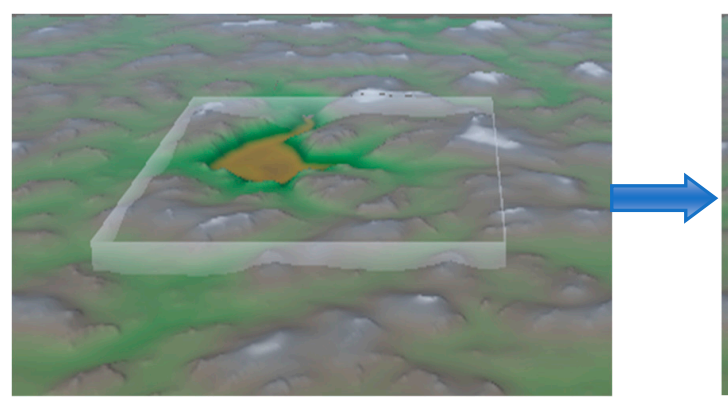

(a)

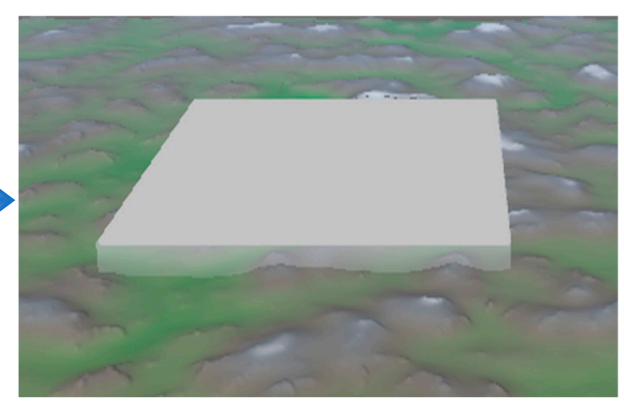

(b)

Figure 3. Demonstration of GMAC Terrain Normalization performed by smart geospatial bi-axial inclinometer nodes (SGBINs) as: (a) normal SGBIN clusters placement; (b) normalization of SGBINs' outputs.

\subsection{Seismic Noise Filtration (SNF)}

The presence of micro-seisms in SP1 constituted a regular data pattern in the seismic instrumentation called stochastic seismic noise that leads to false alarms and poor data integrity. The first step was to skim or extract the seismic noise of the first 500 samples by applying the Savitzky-Golay filter (savgol_filter) in MicroPython as a signal. The savgol_filter (signal, windowsize, order of polynomial) operates at the embedded hardware level. In the Savitzky-Golay filter in MicroPython, the SGBIN data SP1 consists of a time series set of $S\left\{x_{j} y_{j}, t_{j}, t_{j-1}\right\}$ with points $(j=1, \ldots, n)$, where $x$ is an independent variable at $t_{j}$, and $y_{j}$ is an observed value at delayed $t_{j-1}$. They are treated with a set of $\mathrm{m}-\mathrm{xy}$ convolution coefficients, $\mathrm{Ci}$, according to the expression

$$
y_{t}=\frac{1}{h}\left(\sum_{i=-\left(\frac{n p-1}{2}\right)}^{\frac{n p-1}{2}} a_{i} x_{t+i}\right)
$$

In the triangular moving average (TMA), i.e., the function smoothTriangle (noisy signal, degree of filter) at the PC level, two simple moving averages (SMA) are computed on top of each other in order to gain more weight to nearer (adjacent) points. This means that first our SMAi are computed and then a TMA $i$ for the window size $n$ is computed as:

$$
\mathrm{TMA}_{i}=\left(\mathrm{SMA}_{i}+\mathrm{SMA}_{i+1}+\ldots+\mathrm{SMA}_{i+n}\right) / n
$$

The generated filtered time series array (FTSA0) was subtracted from the FTSA of the original signal, i.e., 500 samples from $t 0$ to $t 499$, and difference was saved in the second array called seismic noise array (SNA0).

$$
\mathrm{SNA}=\mathrm{SP} 1-\mathrm{FTSA}
$$

Later on, this SNA was sent to SGBIN as a feedback tuning coefficient to be subtracted from the transmitted signal of SGBIN to reduce the level-2 computation complexity.

$$
\mathrm{SP} 2=\mathrm{SP} 1-\mathrm{SNA}
$$

This technique reduced the filtration cost as well as the expected computation cost of SWEDA in the next phases. 
In Figure 4, we have two plots, seismic signal with noise and filtered signal. We averaged all the data samples from two sites and considered only 10,000 samples. For feature extraction, we had to filter meaningless information out of the 10,000 samples. In a noisy signal, the magnitude of the angles $1.5^{\circ}$ and $-0.5^{\circ}$ are the values that lead to false alarms and wrong detection of seismic events. The correct values are in the range between $0.3^{\circ}$ and $-0.3^{\circ}$, which need to be processed. The kurtosis value and S/L-Kurt algorithm [39] can be used here for microseismic signals.
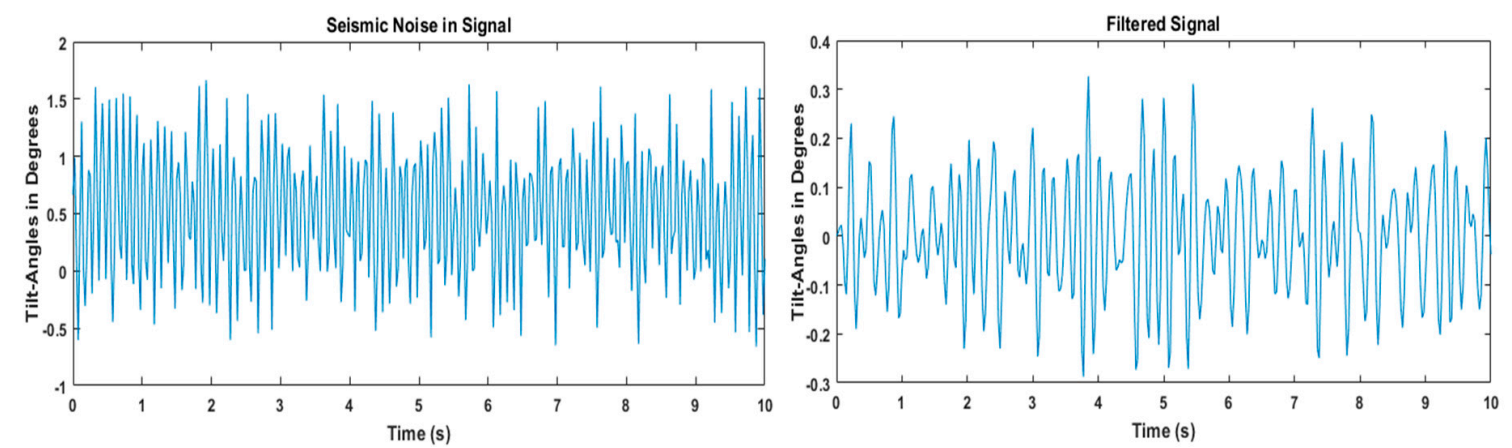

Figure 4. Seismic noise filtration and new refined signal.

\subsection{Peak Detection Sequence (PDS)}

The second step after the FTSA0 and SP2 was detecting the peaks out of real-time values. The peak detection sequence (PDS) used in this work was only based on unique amplitude and frequency detection. The algorithm scanned the unique peaks and frequencies only, i.e., it first searched for the first maximum for early warning, then the second maximum, and accordingly the fifth maximum and eighth maximum.

In Figure 5, the circles show the peaks detected and set as local maxima, and when the next peak came it became local maxima, i.e., only increasing peaks were detected for amplitude variation mapping. If the next tilt angle magnitude was equal or less than the current local maximum, the algorithm ignored it. The purple dotted line was the mean of 500 samples in $10 \mathrm{~s}$. Let us consider that we have $N$ clusters of seismic nodes placed geo-spatially transmitting $D$ datasets of time series as the repository $R(d)$ :

$$
\mathrm{R}(\mathrm{d})=\left\{\begin{array}{l}
N=[N 1, N 2 \ldots N n] \mid N 1=[D 1 a, D 1 b, D 1 c \ldots D 1 n] \\
\text { Where } D 1=\{\text { set of simultaneus data streams of timeseries }\}
\end{array}\right.
$$

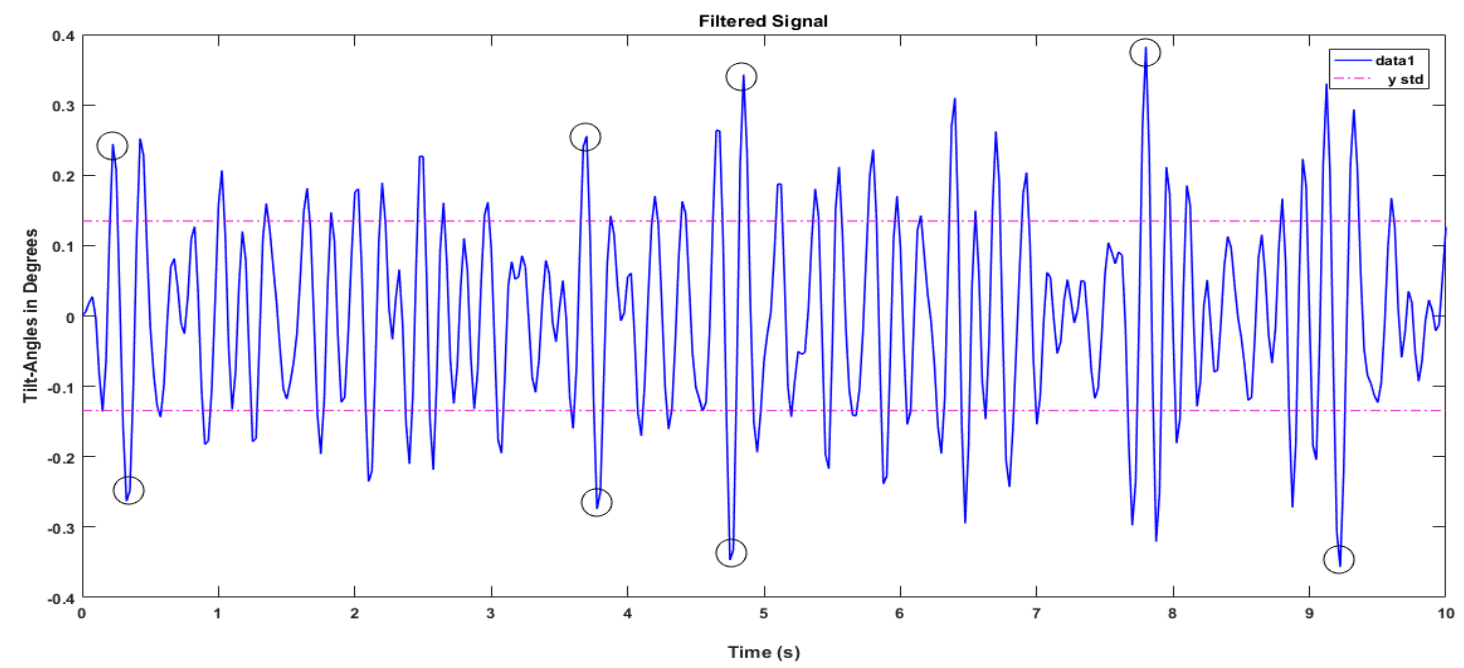

Figure 5. The runtime signal mean calculation and incremental peak mapping sequence. 
In recent works carried out in 2017-2018, time-series data nature detection for stationarity and non-stationarity was tested that increased the delay as well as the computation cost of procedures. If the data was stationary, the power spectral density (PSD) was calculated, and if it was non-stationary, the Hilbert-Huang transform (HHT) was calculated. It was not feasible to calculate PDS and HHT for $\mathrm{R}(\mathrm{d})$ due to computation cost issues. A precise illustration in Table 1 clarifies that any operation that is frequency-transformed and filter-based will cost the most. The Hamming window is the cheapest and most effective algorithm for the real-time computation of time-series signals. Every inclinometer frame was considered, where (i) $\mathrm{M}$ is the number of samples from the 18-bit ADC within a $500 \mathrm{~ms}$ frame, (ii) NA is the next two's power, and (iii) Rfb is the number of the filter banks.

Table 1. The computational cost of the vibration event detection approaches.

\begin{tabular}{ccc}
\hline Algorithms & Computational Cost & Floating-Point Operations \\
\hline Hamming Windowing & $\mathrm{M}$ & 1.440 \\
Fast Fourier Transform (FFT) & $\mathrm{NA} * \log 2(\mathrm{NA})$ & 22.528 \\
$(\mathrm{abs}) 2$ & $\mathrm{NA} / 2$ & 10.24 \\
Filter Bank & $\mathrm{NA} / 2{ }^{*} \mathrm{Rfb}$ & 49.152 \\
Logarithm & $\mathrm{Rfb}$ & 48 \\
\hline
\end{tabular}

\subsection{Autoregressive Pattern Mapping Sequence (APMS)}

The earthquake sequence is first p-wave, then s-wave, and finally, Love wave. From the median of seismic signals, the amplitudes of characteristic waves are scaled and correlated, or the wave window is moved in a sequence with respect to Figure 1. The PDS calculations in Figure 4 were assigned to counters, and each counter contributes the size of windows for APMS.

In Figure 6, the more disrupting wave that arrives first is the p-wave, and its correlation window is represented with the red rectangle. The s-wave has to be checked after that, shown in the green rectangle, and Love waves are presented within the orange triangle. This sequence has to be verified to ensure the type of seismic event and expected fault caused by this measured time series of tilt angles. The magnitudes of tilt angles for the orange rectangle, i.e., Love waves, should be between $-0.05^{\circ}$ and $0.05^{\circ}$. The first window in the figure has values between $0.28^{\circ}$ and $-0.28^{\circ}$, which are the second-highest in the histogram plot.

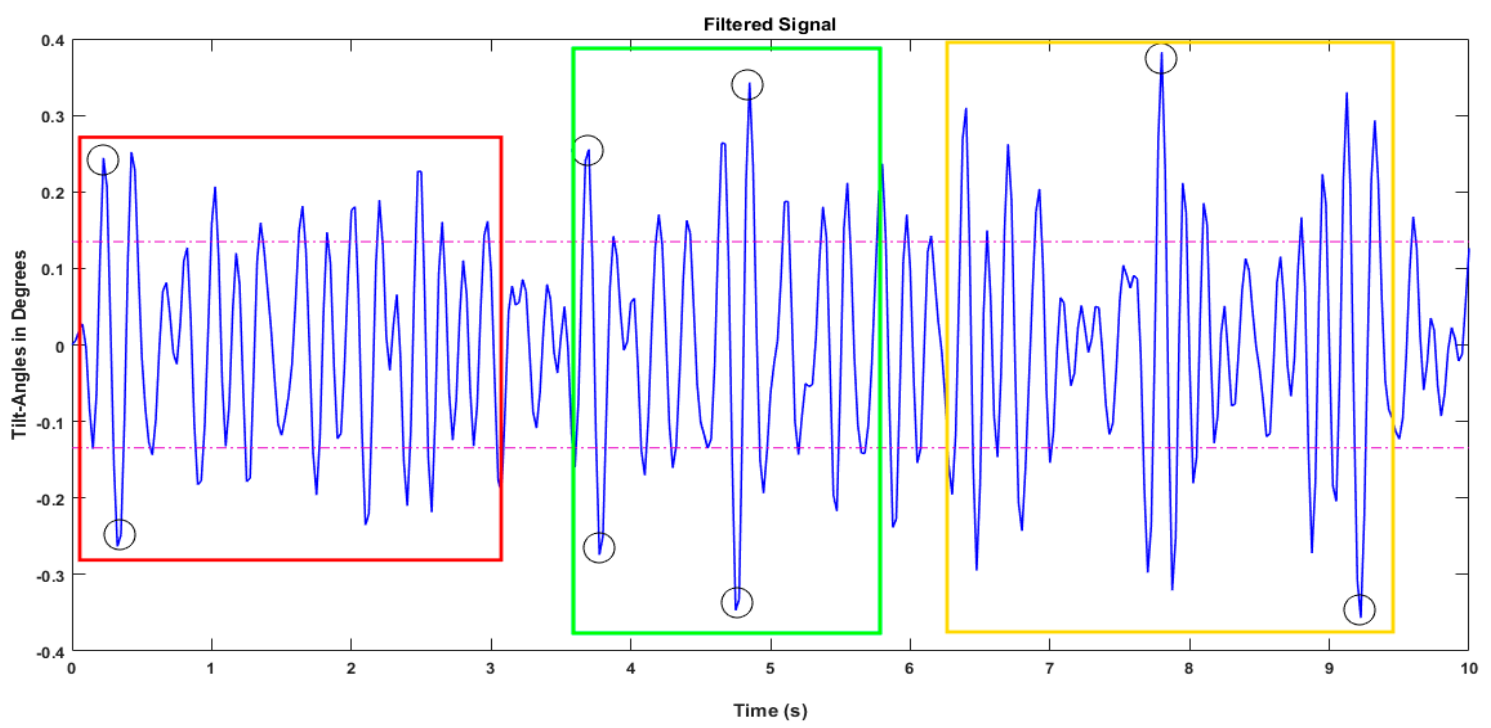

Figure 6. The runtime signal mean-clustering calculation. 


\subsection{Scaling Coefficients Array Generation Sequence (SCAGS)}

It happens $97 \%$ of the times that amplitudes trained for seismic waves windows are not exactly matching the run-time sensor data due to wavelengths, frequency, and amplitudes. The criticality is for the shape of the waves to be matched by dividing the signal into wave windows to measure the ratios for mapping the factor tuning that is only acceptable from 0.71 to 0.95 , and in reality, it never goes above 0.83 .

The variety and volume of peaks observed in Figure 7 show that the superimposed signals show three overlapping criteria: the first with a range between $0.49^{\circ}$ and $-0.49^{\circ} \theta_{1}$ (pk-pk) with a sample space of 7.5-8.8 s, the second with a range of $0.26^{\circ}$ to $-0.26^{\circ} \theta_{2}$ (pk-pk) and a sample space of 5-6.2 s, and the third with a range between $0.42^{\circ}$ and $-0.42^{\circ} \theta_{3}$ (pk-pk) and a sample space of 2.5-3.8 s. These factors constitute an accurate calculation of the STA/LTA parameters estimation. Most of the clusters of the peak-to-peak angles are dense and high in quantity, more than the STAs and overall LTAs. SCAGS reflects the resemblance percentage or coefficient of similarity.

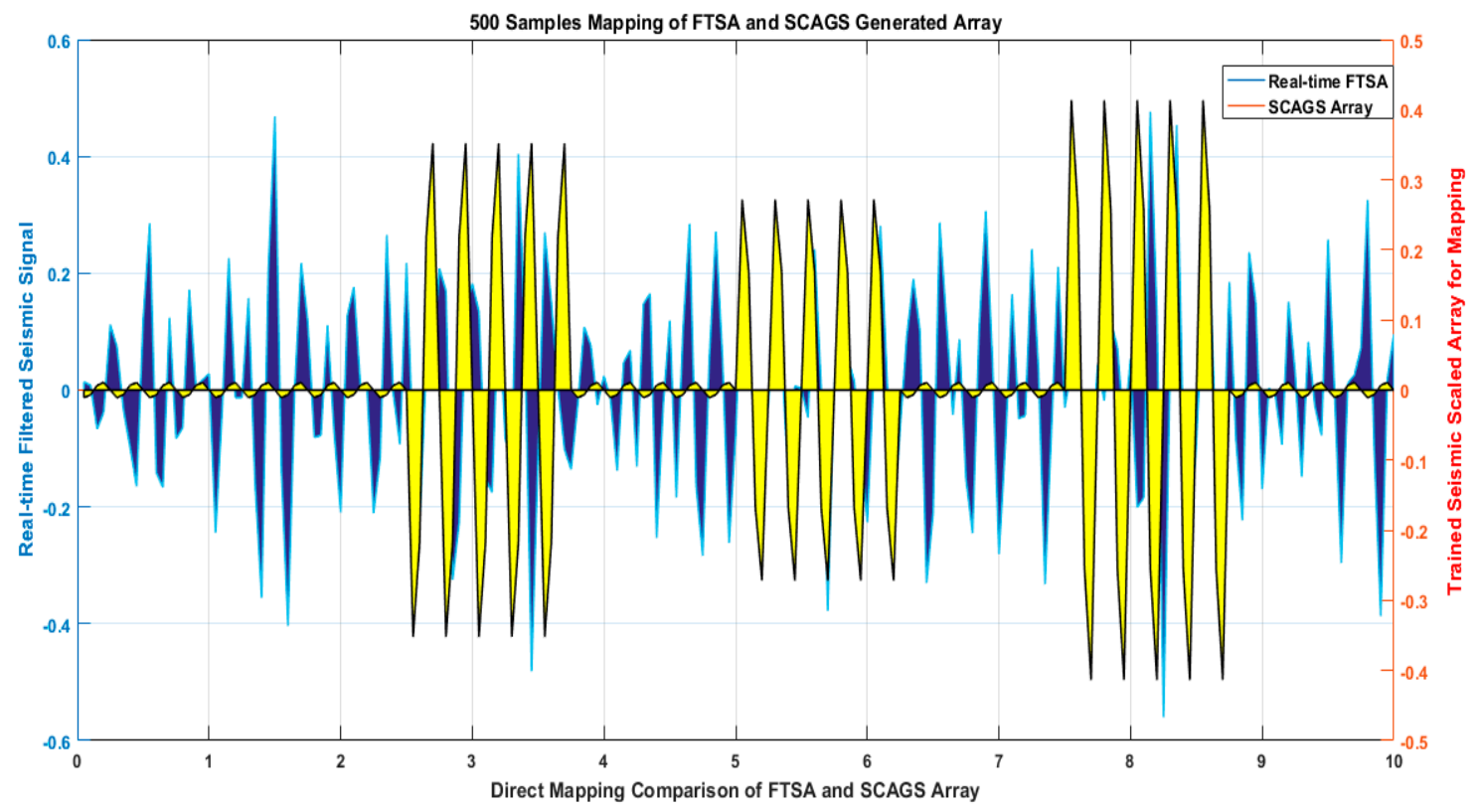

Figure 7. The scaling coefficients array generation sequence (SCAGS) mapping for SP2.

\subsection{STA/LTA Arrays Windows Sequence (SLAWS)}

The application of STA/LTA arrays is the smartest way for automated recording of critical events. Most of the unique samples or events are traced using STA, and the complete sequence with heterogeneous events is tracked using LTA. The STA/LTA has key initiation parameters that must be automated for non-stationary anomaly events like earthquakes.

Different seismic frequencies were observed in different earthquakes over the course of time. Let the standard alarming seismic waves frequencies be $\mathrm{f}_{S T A N D A R D}>0.1 \mathrm{~Hz}$ and onwards. Let us generalize that the $\mathrm{P}$-wave has a frequency between 1 and $16 \mathrm{~Hz}$ generalized from [42], the S-wave can be [43,44] generalized as $0.1-8 \mathrm{~Hz}$, and both [45] R- and L-waves have $0.01-2 \mathrm{~Hz}$ at maximum. STA and LTA have the following generic event detection parameters (EDP):

- $\mathrm{W}_{\mathrm{d}}$-Waveform data ('shm_thingspeak.csv')

- $\quad \mathrm{F}_{\mathrm{s}}$-Sampling frequency (as per Nyquist criterion, i.e., $\left.2 \mathrm{~F}=2(1 \mathrm{~Hz}), 2(1.5 \mathrm{~Hz}), \ldots 2(24 \mathrm{~Hz})\right)$

- $\quad \mathrm{L}_{\mathrm{ts}}$-Length of time series (80,000 samples)

- $\mathrm{T}_{\mathrm{v}}$-Time vector of the waveform (10,000 samples)

- $\mathrm{ABS}_{\mathrm{ts}}$-Absolute value of time series $(40,000)$

- $\mathrm{W}_{\mathrm{STA}}=\mathrm{EDP}_{1}{ }^{*} \mathrm{~F}_{\mathrm{S}}-\mathrm{STA}$ window size $($ Dynamic) 
- $\mathrm{W}_{\mathrm{LTA}}=\mathrm{EDP}_{2}{ }^{*} \mathrm{~F}_{\mathrm{S}}-\mathrm{LTA}$ window size $($ Dynamic $)$

- $\quad W L_{L T A}=W L_{L T A}-$ current length of growing LTA window (sizes of the time axis of respective wave)

- $\quad \mathrm{TR}_{\mathrm{ON}-\mathrm{STA}+\mathrm{LTA}}=\mathrm{EDP}_{3}-$ trigger on when STA_to_LTA exceeds this threshold

- $\quad \mathrm{TR}_{\mathrm{ON}-\mathrm{STA}-\mathrm{LTA}}=\mathrm{EDP}_{4}-$ trigger off when STA_to_LTA drops below the threshold

In our case with four wave types in one sequence, we will have three STAs and one LTAs array for a single sequence of events as exhibited in Figure 8.

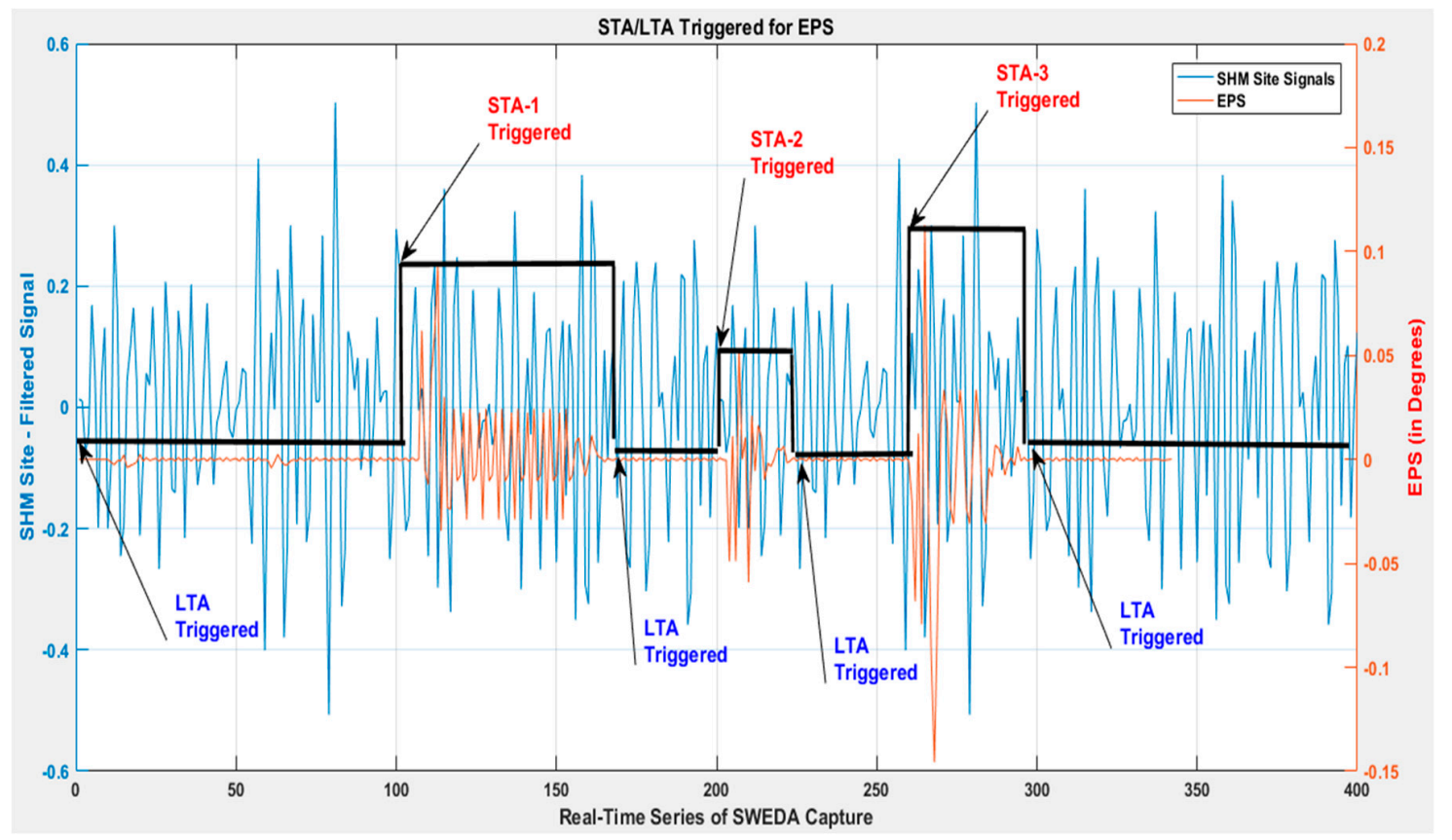

Figure 8. The automated triggering of short-time average over long-time average (STA/LTA) with SWEDA.

\subsection{Earthquake Probabilistic Sequence (EPS)}

The EPS condition can only be satisfied under triggering conditions: $\mathrm{STA}_{1}=\mathrm{P}$ wave, $\mathrm{STA}_{2}=\mathrm{S}$ wave, and $\mathrm{STA}_{3}=$ Surface wave in $\mathrm{LTA}_{1}, \mathrm{LTA}_{2}$, and $\mathrm{LTA}_{\mathrm{N}-1}$, provided that rations of STAs defined as $\mathrm{R}_{\mathrm{STAs}}$ is in the range of $0.8-1.2$ and $R_{\text {LTAs }}$ is in range of 0.7-1.3. EPS is shown in Figure 1.

\subsection{Seismic Feature Extraction (SFE)}

After the waves and earthquake detection estimation of velocities, the next step is towards an early warning and fault prediction. Refer to [17] for equations for angles, displacement, and velocities.

\subsection{Event-Triggered ADC Scaling/Range and Communication Bus Configuration}

The final step after the event detection is to optimize the ADC resolution according to the feature extraction requirements. Increasing the resolution and range will make the ADC flexible for STA/LTA recorders and thus result in the need for the increased data rate to accomplish the complete event detection and characterization milestone. The tiltmeter nodes in Figure 10 are the selected nodes which are standard industry nodes with (CAN in Automation) CiA-301 compliance. The PDS and STA demand will trigger an increase or decrease in ADC resolution, and LTA will trigger the service data object (SDO) parameter 0x20F2 and 0x20F3 in byte 5 of the CANopen packet. The ideal node for this application is the one with the reserved command for ADC configuration, i.e., scaling, resolution, range, and sampling rate.

Figure 9 interprets the SWEDA process tested in MATLAB that had data in binary format and functions that interchanged binary data as arguments and return types. OMPC [42] and LiberMate are 
the two packages that convert MATLAB functions to Python for hardware implementation, and very fruitful output has been shown in the results sections.

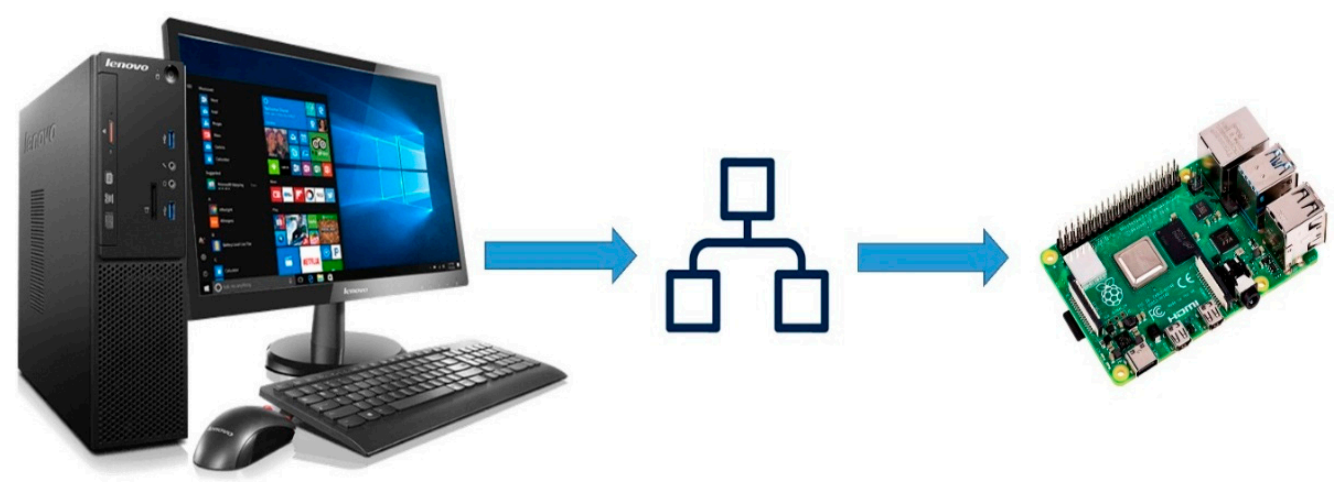

Figure 9. MATLAB-Raspberry Pi 4 Implementation using OMPC and LiberMate

\section{Smart Geo-Spatial Inclinometer Nodes Application Design}

The smart geospatial inclinometer nodes application designs (SGSINs) fill the gap of programmable resolution ADC, sampling rate, and communication for input- and output-tuning for NDT and NDE assessment procedures. In our research project, we designed and fabricated two SGBINs as:

- $\quad$ Flat SGSINs with two accelerometers used as inclinometer sensors (F-SGSINs)

- Cylindrical SGSINs with $2+N$ sensor support (C-SGSINs)

CANopen was used as a communication interface and a common bus. The details of these nodes are given in their respective sections. The two nodes are exhibited in Figure 10.

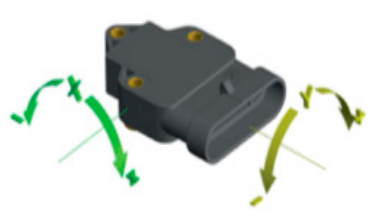

(a)

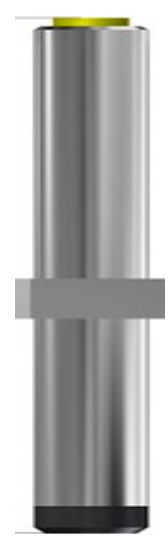

(b)

Figure 10. The physical appearance of SGBINs as (a) F-SGBINs and (b) C-SGBINs.

It is noteworthy to highlight that the SGBINs displayed in Figure 10 are enhanced with remotely programmable and configurable parameters using CANopen.

\subsection{Flat SGSINs}

The block diagram of the SGBIN version 1 is given in Figure 11, focusing on the measurement constraints for SWEDA explained in Section 2. A bi-axial accelerometer ADXL203 was used for acceleration as well as inclination measurements. 


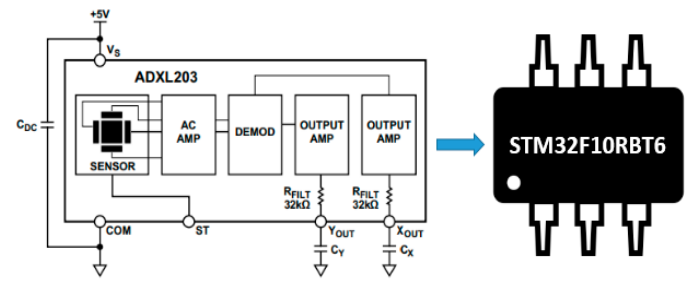

(a)
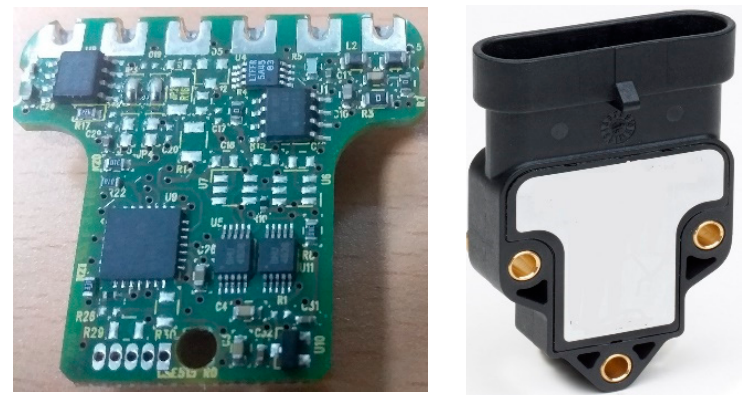

(b)

Figure 11. The F-SGBIN views as (a) F-SGBIN architecture; and (b) F-SGBIN fabrication.

In Figure 10, the ADXL203 was interfaced with an STM32F10RBT6, i.e., 32-bit microcontroller with a 12-bit ADC consisting of two channels with a sampling rate of 1 us and a CAN-Open transceiver (a), and the PCB, as well as the IP68 enclosure, is displayed in (b).

\subsection{Cylindrical SGBINs}

An additional 24-bit programmable sigma-delta ADC was used with a 10X programmable gain amplifier (PGA) to increase the resolution of the signal as per run-time requirements. A $32 \mathrm{MB}$ flash memory was also added for more mathematical operations for more sensors at the node level. A very comprehensive block diagram of C-SGBIN is presented in Figure 12.

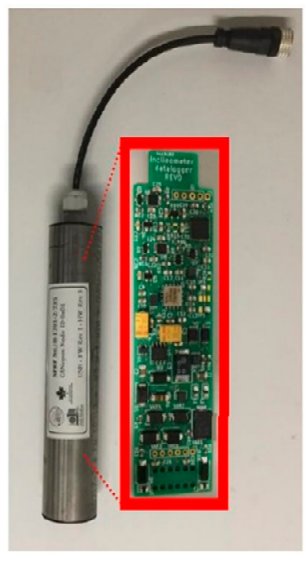

(a)

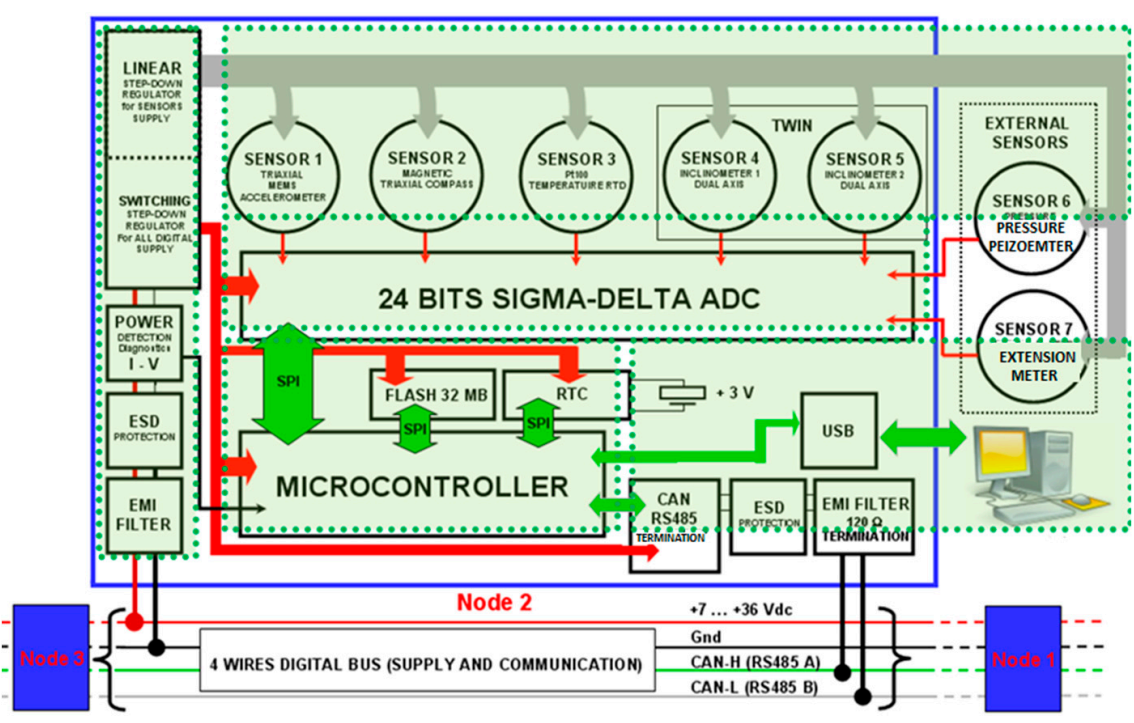

(b)

Figure 12. The C-SGBIN views as (a) C-SGBIN fabrication and (b) F-SGBIN architecture.

In Figure 12, the C-SGBIN has seven sensors that can be utilized by relevant algorithms for constraint configurability. This node is specialized in underground monitoring and has IP68 protection.

The ground motions associated with $\mathrm{P}, \mathrm{S}, \mathrm{R}$, and L seismic waves required a specialized topology of sensors for minimum computation cost using matching amplitudes of direction and angular displacements shown in Figure 13. 


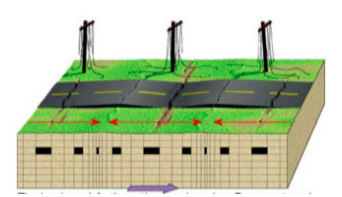

(a)

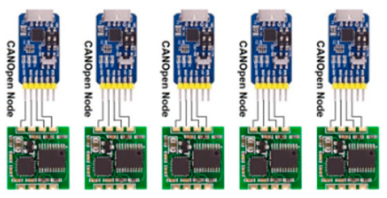

(e)

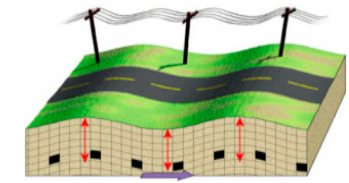

(b)

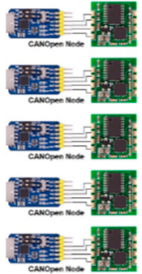

(f)

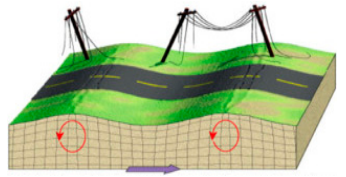

(c)

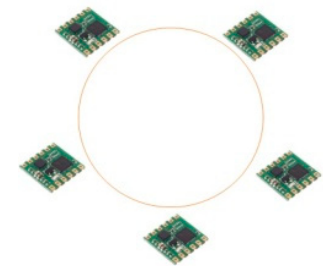

(g)

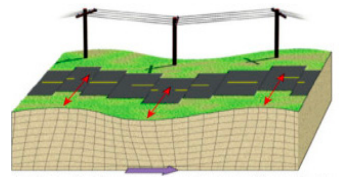

(d)

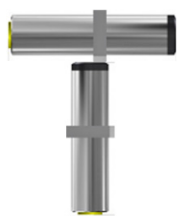

(h)

Figure 13. Realization of a SGBIN cluster topologies according to seismic wave ground motions as (a) P-wave ground motions; (b) S-wave ground motions; (c) R-wave ground motions; (d) L-wave ground motions; (e) SGBIN topology for P-wave detection; (f) SGBIN topology for S-wave detection; (g) SGBIN topology for R-wave detection; (h) SGBIN topology for L-wave detection.

\section{Case Study: Experiment Designed and Implemented at Qatar University}

The chosen case study was the SHM system for Qatar University (QU) as a potential SWEDA-SHM study ground. Three different specifications and configuration systems were installed at QU at two physically $1 \mathrm{~km}$ apart unique locations, namely, SHM-QU-B09 Lab and SHM-QU-CO5-Bridge. The SWEDA-SHM system details for these locations are:

- $\quad$ SHM-QU-CO5-Bridge SWEDA-SHM Site (SHM-BS) System with 5 F-SGBINs.

- $\quad$ SHM-QU-B09-Lab SWEDA-SHM site (SHM-LB) with 5 F-SGBINs and 10 C-SGBINs.

The entire deployment plan for the case study at QU is shown in Figure 10, published in our earlier papers.

In Figure 14, a very basic regional-level SWEDA-SDM hardware prototype is presented, i.e., the two unique remote blocks were connected with the SHM system called out-surface board (OSB) with IoT node software using the CANopen network with a CAN-USB adapter by Gingko. The CANopen addresses were 1, 2, 3, 4, 5 on both sides. The two SHM site nodes were connected to the I/O multiplexer block of the SWEDA-SHM gateway (installed with IoT gateway software) with a keyboard-video-multimedia (KVM) switch for multiple displays, communication ports, and a power supply unit (PSU). The term RSS refers to the resilience support system in Figure 14, which means that the early warning and seismic event detection results are used by a resilience support system to alarm inhabitants to be aware and ready to cope with the situation. PRCL1 is the private cloud server that assists the PC-level computations like PDS sample streams to overlap the run-time seismic signals. There can be more than $9999999+$ seismic samples overlapped over the run-time SP2 to compute the ratio as a similarity index between 0.1 and $\sim 0.99$.

In Figure 15, the physical deployment of the SWEDA-SHM-QU case study is displayed as C05-Bridge (yellow) and B09-Lab (cyan). 


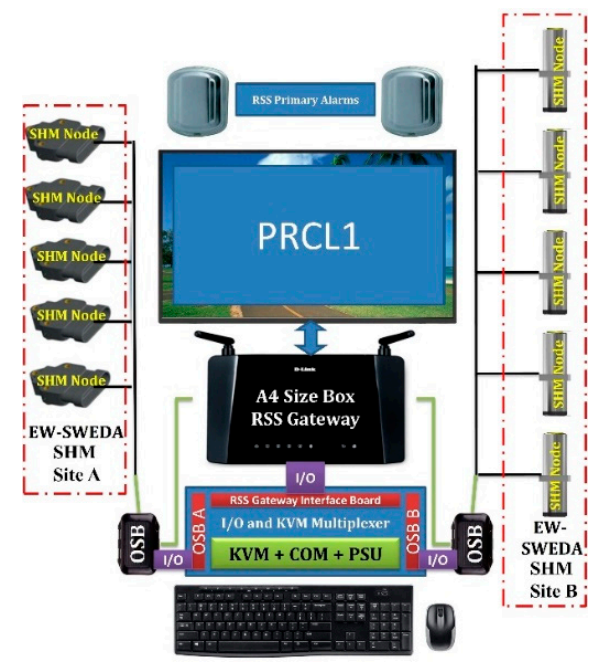

Figure 14. Realization of a regional SWEDA-SDM hardware prototype.

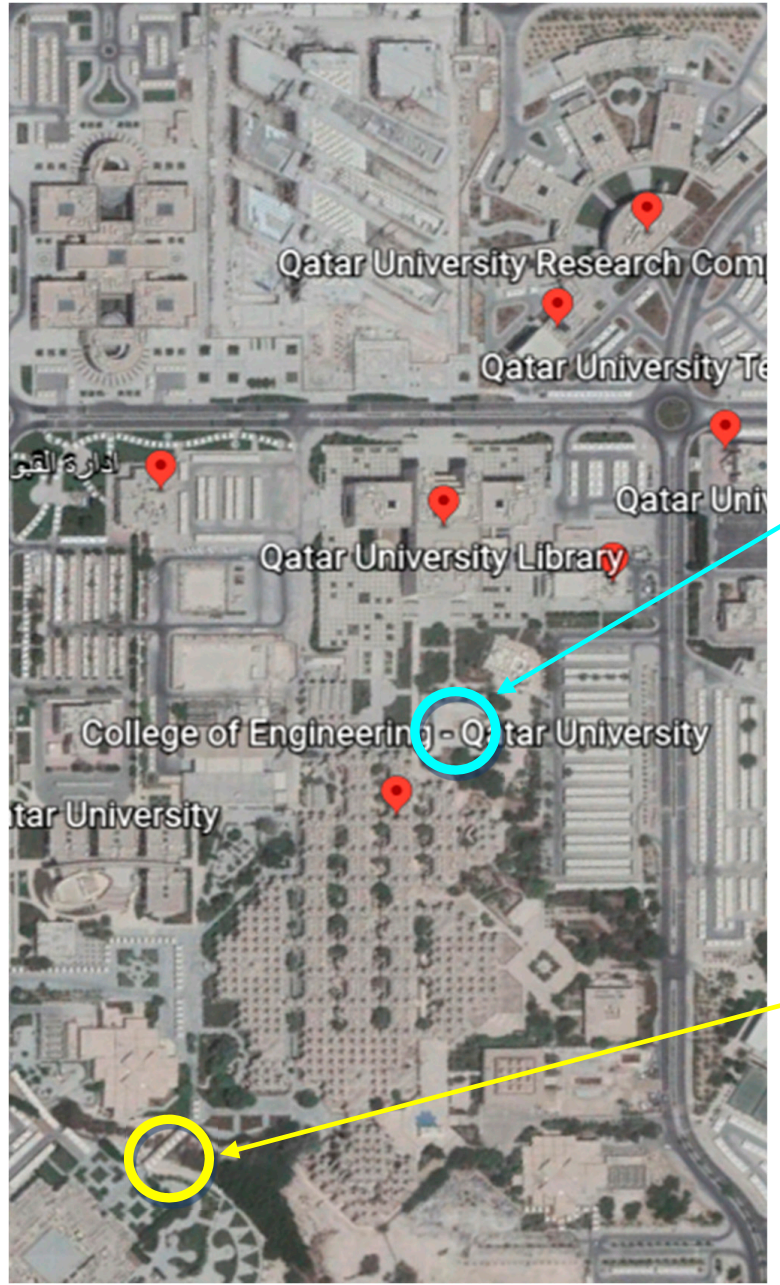

(g)

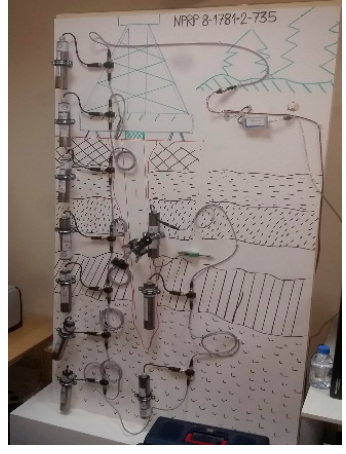

(a)

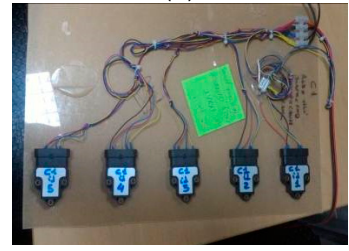

(b)

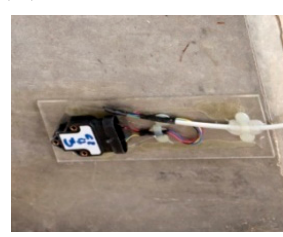

(d)

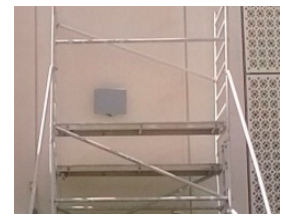

(e)

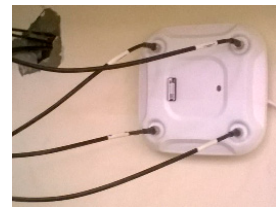

(f)

Figure 15. Case study: Qatar University (QU) SWEDA-SHM sites deployment details listed as (a,b) B09 Lab with 10 C-SGBINs on SHM stand and 5 F-SGBINs on table and (c) out-surface board (OSB) and a F-SGBINs at the QU Bridge Site; (d) F-SGBINs at QU Bridge Site; (e) Outdoor Data Unit (ODU) for QU Bridge site mounted on wall of C05; (f) Indoor Data Unit (IDU) for QU Bridge site mounted on wall of C05; (g) overview on Google Maps for QU SHM sites. 
The core challenges in early warning and seismic wave events detection to be resolved at campus-level implementation in this work were:

1. SGBINs placement anomalies, i.e., all sensors were not symmetric neither vertically nor horizontally.

2. SGBINs output contained unwanted amplitudes and frequencies that were increasing the computation costs as well as false detection.

3. The early warning estimation was a major challenge, i.e., the requirement of peak detection or extremities in the signal.

4. The seismic waves automated recording and processing activation needed unique peaks and frequencies identification.

5. The seismic waves runtime similarities estimation with an expected earthquake or seismic signal needed magnitude ratios as well as signal clustering.

6. The STA/LTA triggering only for featured events was a mandatory step that had to be performed.

7. A probabilistic sequence for an earthquake for runtime conditions was also needed for the next or upcoming signals similarity assessment.

8. Sending a new sampling scheme for the optimization of ADC was also needed to only capture the needed signals and reduce the post-analysis costs.

The solution of all these difficulties is given in the results section step by step with an analysis.

\section{Results}

Two identical SHM systems with two heterogeneous node sets, each was used at two different geo-locations as discussed in Section 2. The transmission from the SHM nodes to the gateway was started, and the packets $\mathrm{P}_{\mathrm{N}}$ were sent by the SGBINs to gateway(from Equation (6)).

In Figure 16, a very rich source of data with 16,00,000 x-axis and y-axis tilt angle samples has been shown, with each dataset containing 80,000 samples. These data have been collected from our high-precision nodes placed at two geospatial positions, i.e., Lab106A and C05 Bridge Site at Qatar University, Doha. Two clusters of two tiltmeter nodes with specifications given in Figures 10-12 were networked and their data were collected in our database. For a detailed analysis, data of only two nodes with $\mathrm{x}$ and $\mathrm{y}$-axis tilts were included.
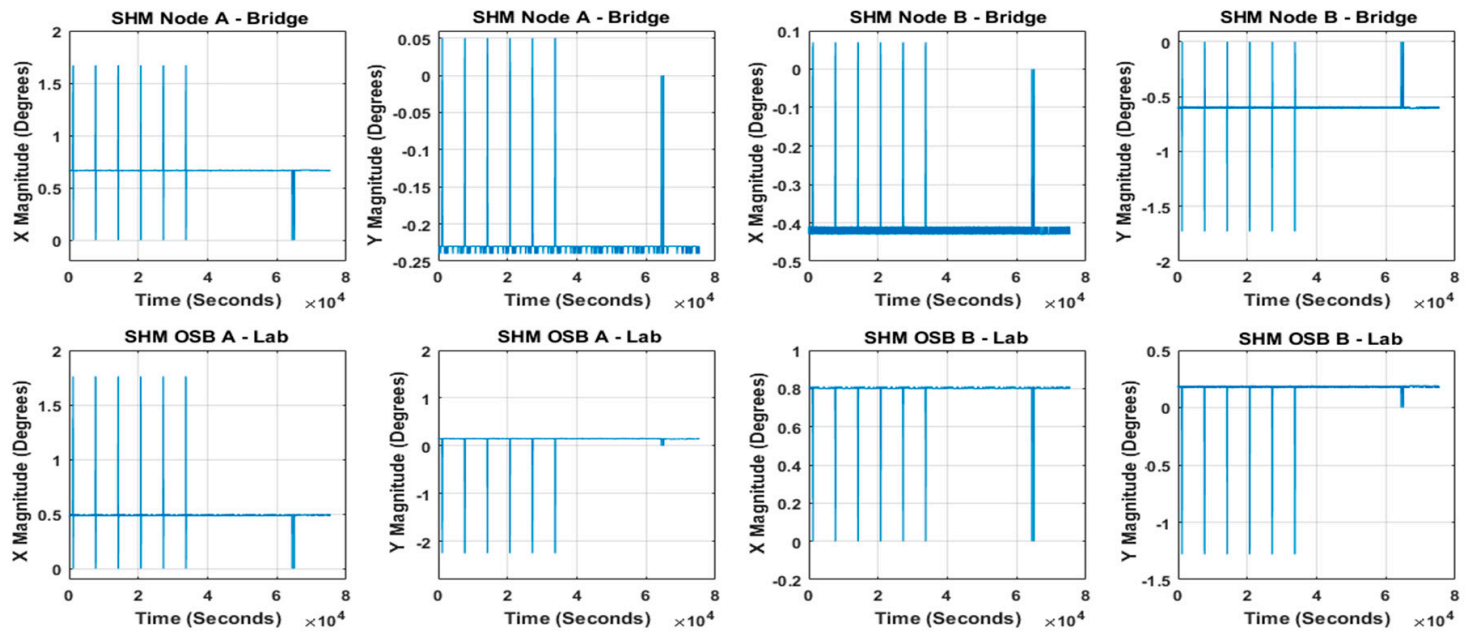

Figure 16. Bi-axial tilt angles data gathered at two unique geolocations in five calendar months $(80,000$ samples).

The data in Figure 17 will probably give false alarms as it has off-sets, we can see that data-centered at $\left(0.23^{\circ},-0.23^{\circ}\right)$ in $\mathrm{x}$-axis and $\mathrm{y}$-axis for node $\mathrm{A}$ at bridge, $\left(-0.39^{\circ},-0.61^{\circ}\right)$ in $\mathrm{x}$-axis and $\mathrm{y}$-axis for 
node $\mathrm{B}$ at bridge, $\left(0.5^{\circ},-0.07^{\circ}\right)$ in $\mathrm{x}$-axis and $\mathrm{y}$-axis for node $\mathrm{A}$ lab, $\left(0.8^{\circ}, 0.23^{\circ}\right)$ in $\mathrm{x}$-axis and $\mathrm{y}$-axis for node A lab at bridge. In Figure 13, we can see that SWEDA had used the relative gradient method to programmatically calibrate all the axis to $0^{\circ}$ to avoid false alarms and ensure accurate performance of micro-steps otherwise all the calculation is useless.
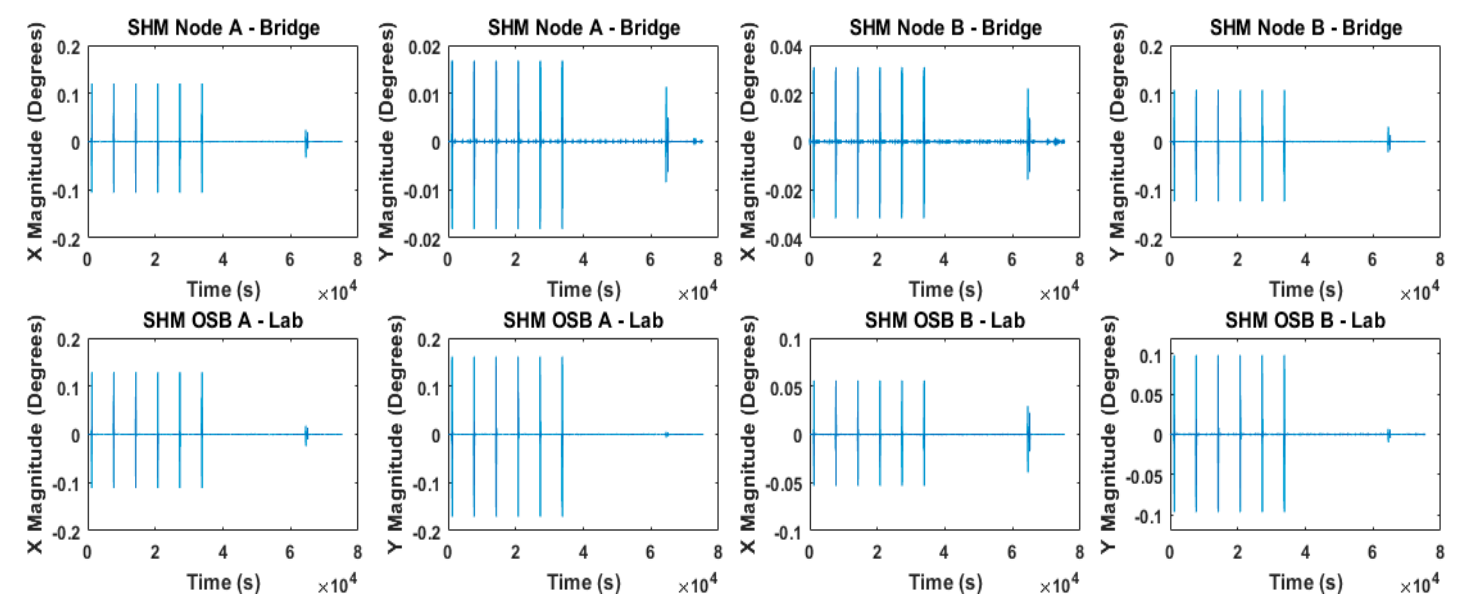

Figure 17. Bi-axial tilt angles data gathered at two unique geolocations in five calendar months $(80,000$ samples).

Furthermore, various data processing techniques like averaging, mean, frequency analysis and histogram. Focusing on the embedded devices for minimum computation cost histogram was selected.

In Figure 18, the histogram of the entire dataset is shown. The most frequent tilt angle values (MFTAVs) on SHM Node A-Bridge were an $\mathrm{x}$-axis tilt angle of $0.67^{\circ}$ and a y-axis tilt angle of $-0.23^{\circ}$. The SHM Node B-Bridge had an $\mathrm{x}$-axis tilt angle of $-0.42^{\circ}$ and a y-axis tilt angle of $-0.61^{\circ}$. SHM OSB A had an $x$-axis tilt angle of $0.51^{\circ}$ and a y-axis tilt angle of $0.295^{\circ}$. SHM OSB B had an $x$-axis tilt angle of $0.8^{\circ}$ and a y-axis tilt angle of $0.24^{\circ}$. Furthermore, the results of various data processing techniques, like averaging, mean, frequency analysis, and histogram, are shown. Focusing on the embedded devices for minimum computation cost, a histogram was the least costly operation. The most frequent values are automatically assigned to thresholding counters in SWEDA. MFTAV contributes to LTA triggering due to the maximum occurrence in the dataset.
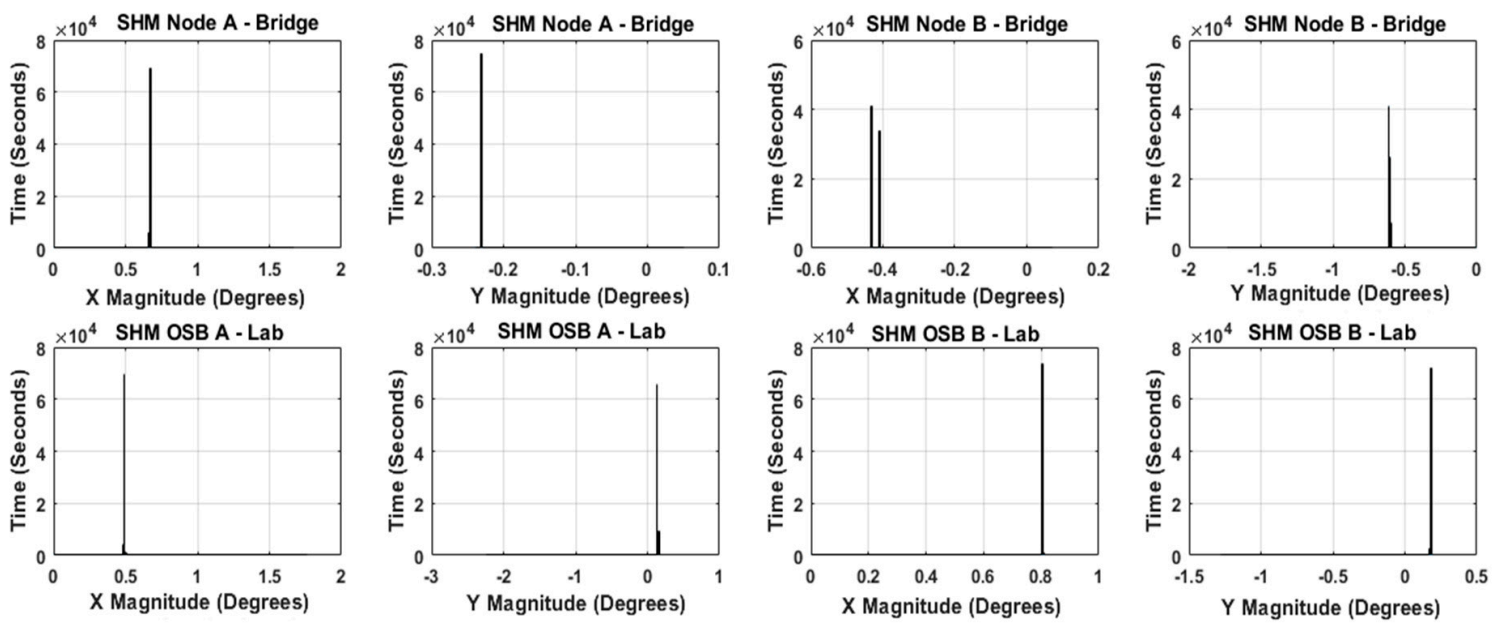

Figure 18. Histogram of data gathered at 2 unique geolocations in 5 calendar months (80,000 samples).

Figure 19 shows the effectiveness in reducing the computation cost of SWEDA in real sense for embedded systems by using the histogram after gradient which shows that the population of zeros is 
very favorable for floating-point operations by resource-constrained hardware like field-programmable gate arrays (FPGA), system-on chip (SoC), application-specific integrated circuits (ASICs), Arduino, and Raspberry Pi 3. SWEDA enabled 62,000 zeros that improved the real-time efficiency by $77.5 \%$ $(62,000 / 80,000)$ in any domain, be it time or frequency.
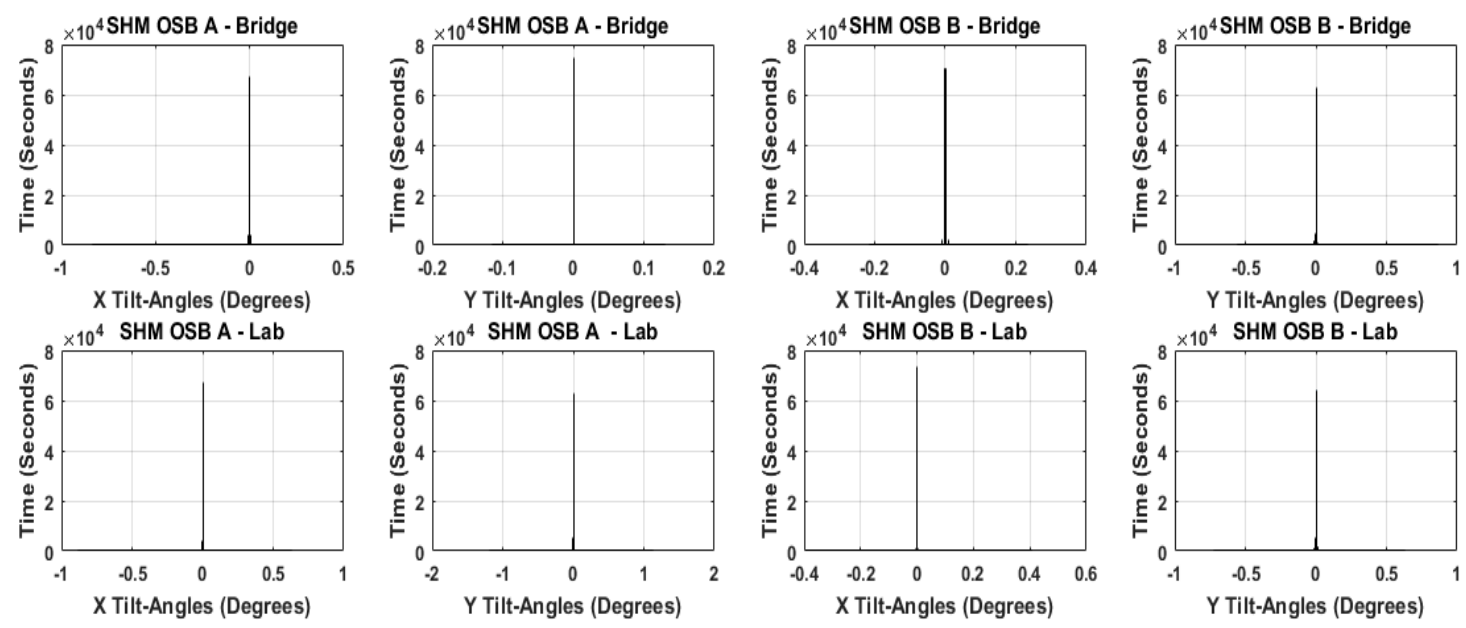

Figure 19. Histogram of data gathered at two unique geolocations in five calendar months (80,000 samples).

In Figure 20, the acceptable range of frequencies is shown that assisted the switching bandwidth of our Butterworth band-pass filter with a lower limit of $2 \mathrm{~Hz}$ and an upper limit of $48 \mathrm{~Hz}$ for optimized feature extraction in SWEDA. SWEDA uses Nyquist criteria that are at least twice the actual frequency of the signal. For detailing, SWEDA operated at five times the frequency of the node signals. The efficiency of the FFT was also increased by $77.5 \%$.
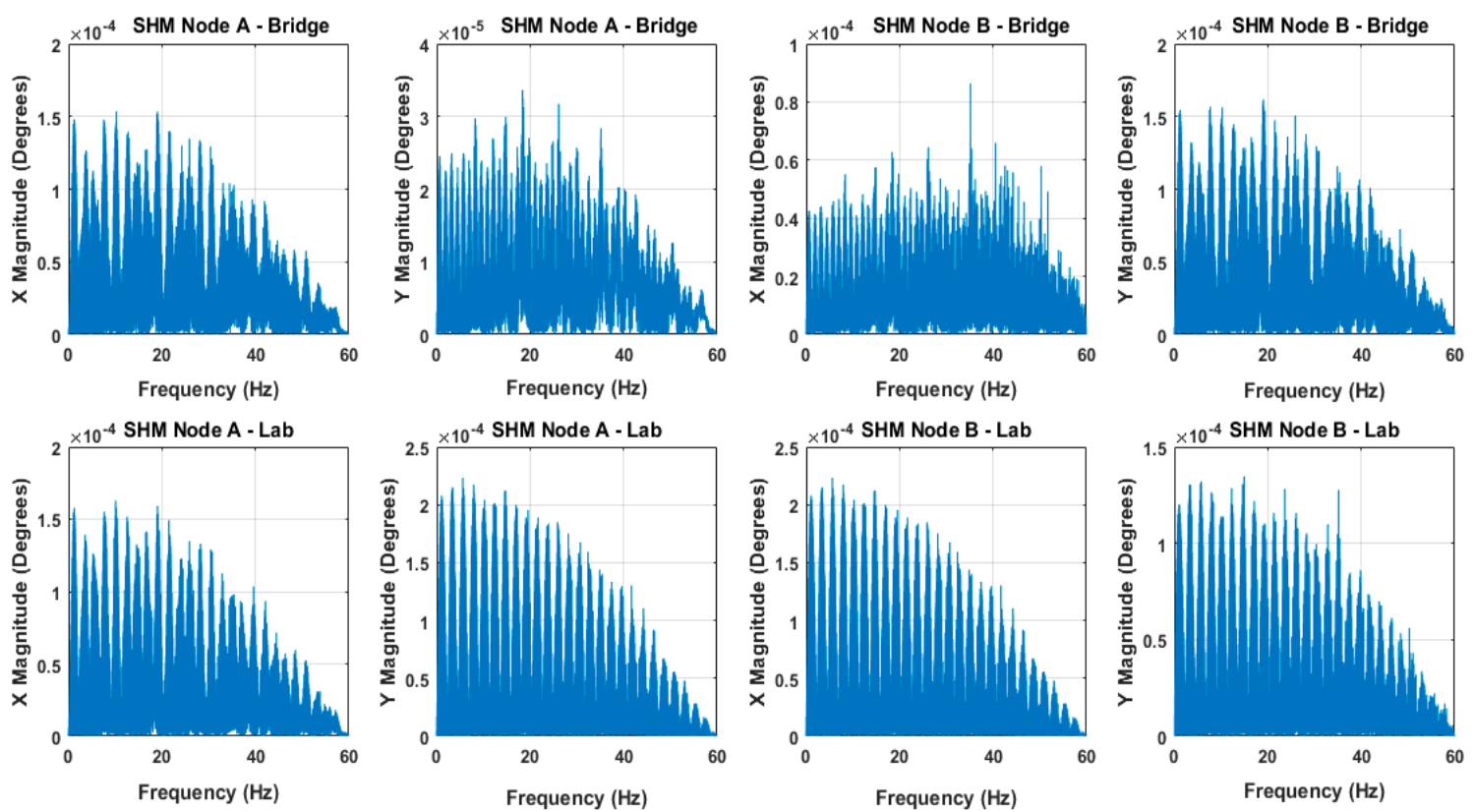

Figure 20. FFT of data gathered at two unique geolocations in five calendar months (80,000 samples).

Figure 21 portrayed the performance of second-order bandpass Butterworth filter with gradient correction, seismic noise filtration, and FFT verification. 

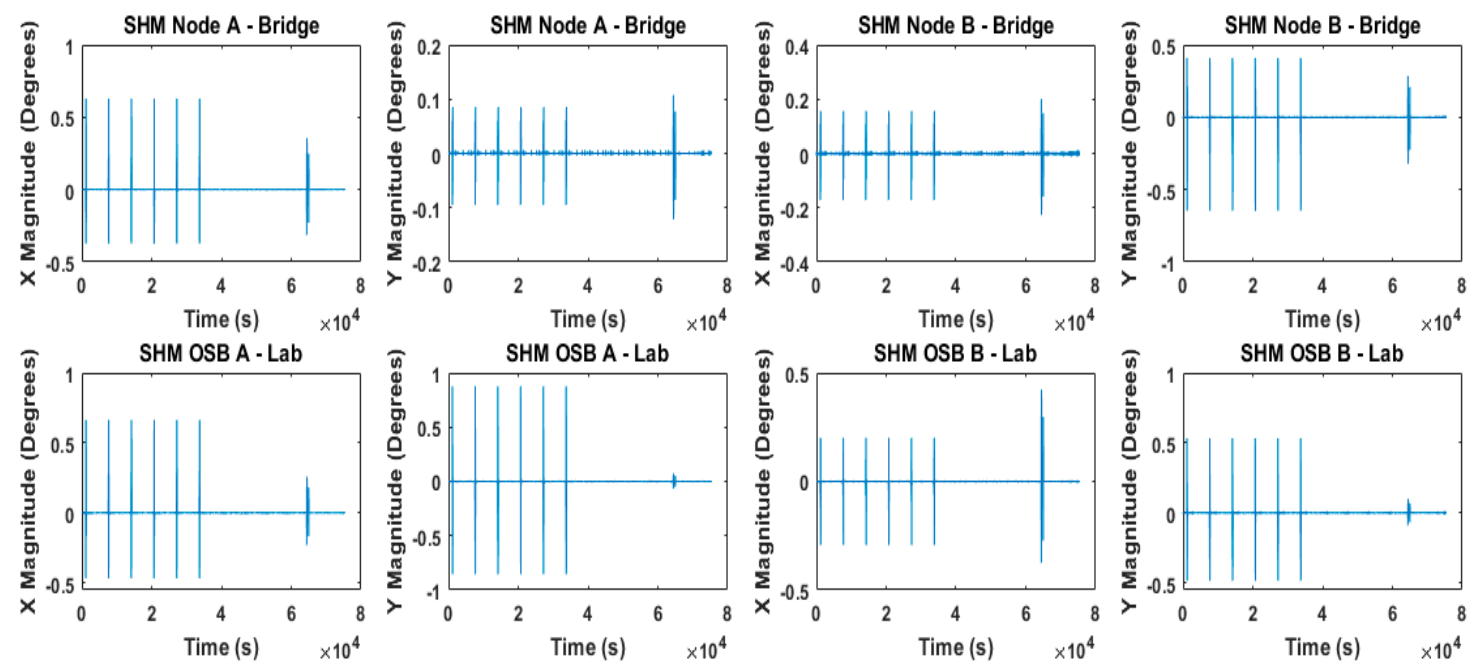

Figure 21. Filtered signals with Butterworth (band-pass) filter (order $=2$ ) for two sites.

The processed data set after the filter is given in Figure 16. After running the PDS and APMS functions in Figure 22 dataset, SWEDA ran the SCAGS loop to store unique structured wave patterns. SCAGS detected only six unique waves that it searched in the STA window. Wave patterns increase the probability of percentage similarity with a dynamically generated EPS.
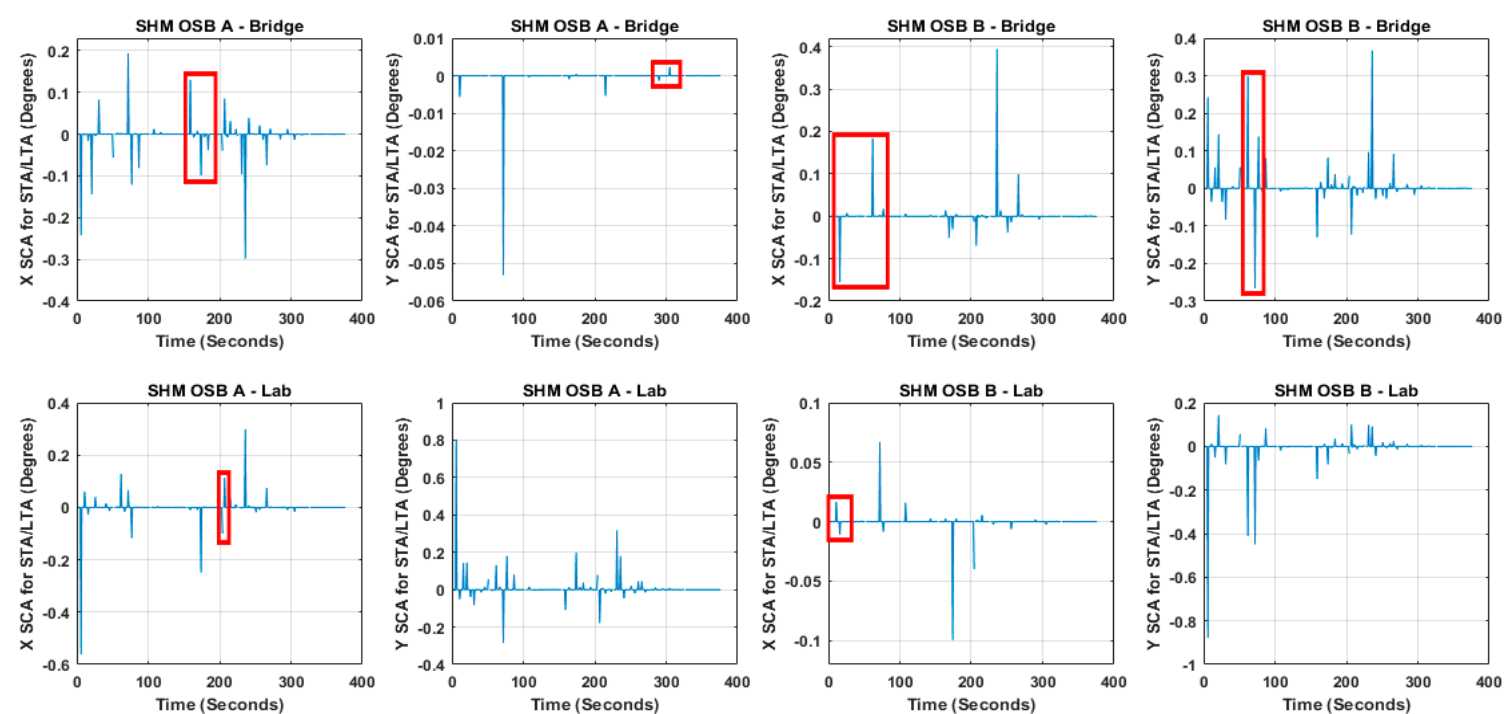

Figure 22. Peak detection sequence (PDS)/autoregressive pattern mapping sequence (APMS) for SCAG for extracted signals for two sites (structured waves mapped $=6$ ).

In Figure 23, a 3D spectrogram is shown. It gives a clear picture of the cross verification of outputs of SWEDA. The orange shade shows the maximum strength of our gradient signal is near 0 , as interpreted by SCAGS. In Figures 16 and 17, it is very obvious that the 0.2 peak-to-peak distance is the least re-occurring cluster which is proof of a seasonality impact in our SHM data set shown in the yellow legend. The blue legend is scattered in the form of small segments and regular intervals, showing the stationarity in the data set. 

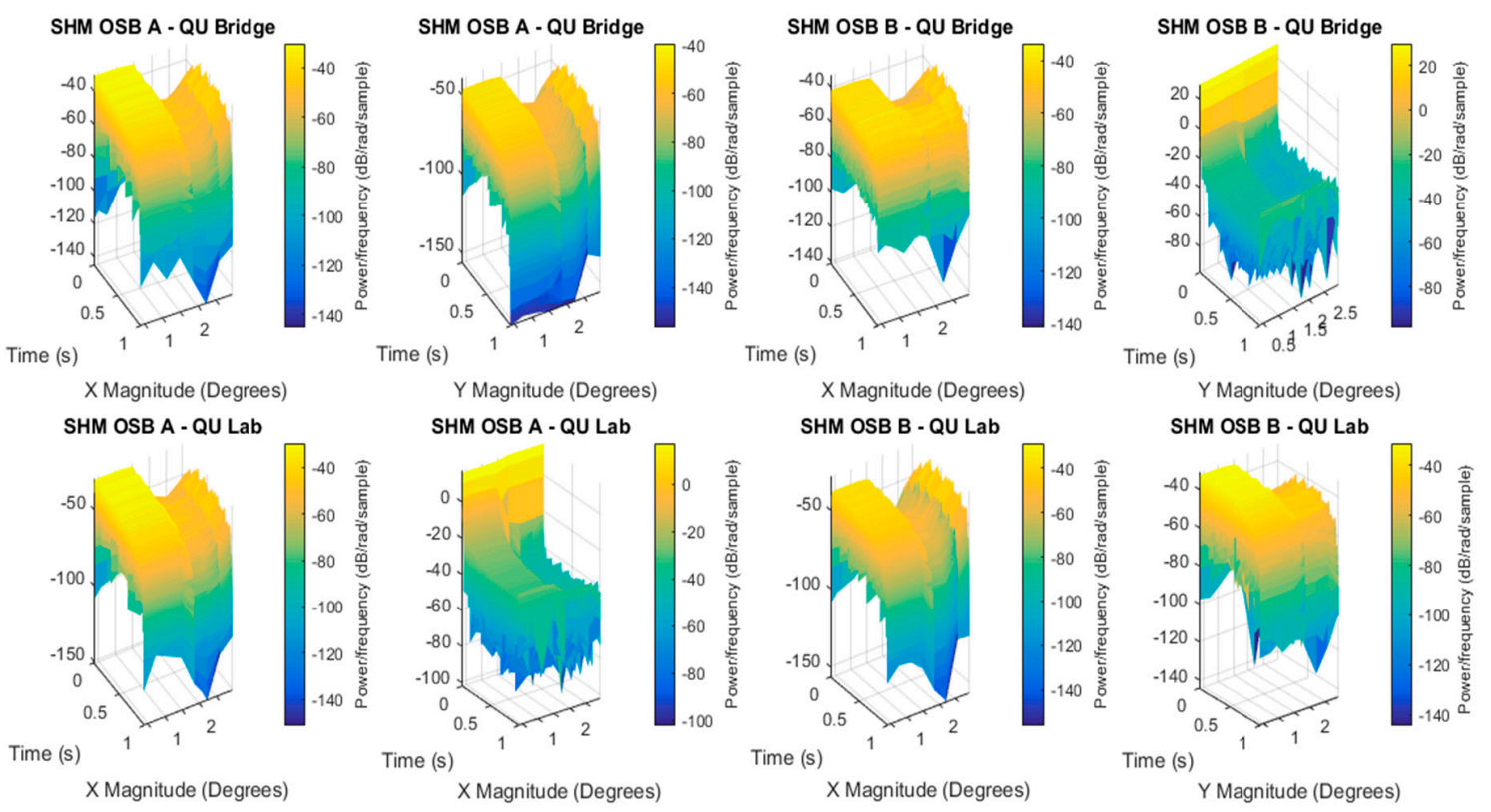

Figure 23. 3D spectrograms in the time domain from two SHM sites (QU Bridge and QU Lab).

In Figure 24, the final output of SWEDA is displayed for the QU bridge. Every time, SWEDA generated a different EPS for the different nodes at runtime to demonstrate its live characterization capability given as a red line. The dataset being highly stationary and only LTAs existed due to the seasonality impact made it very challenging to map STAs for $\mathrm{P}, \mathrm{S}$, and surface waves, even though as per live data it generated its EPS separately for separate data streams from the sensors. The EPS values $(0.09,0.00,0.05,0.02)$ show that for the current situation the percentage resemblance with seismic waves was 0.00 as there was no STA detected from the six waves stored in the SCAGS. However, due to the LTA, there is an extremely negligible chance of an earthquake which can be stated as "no chance".
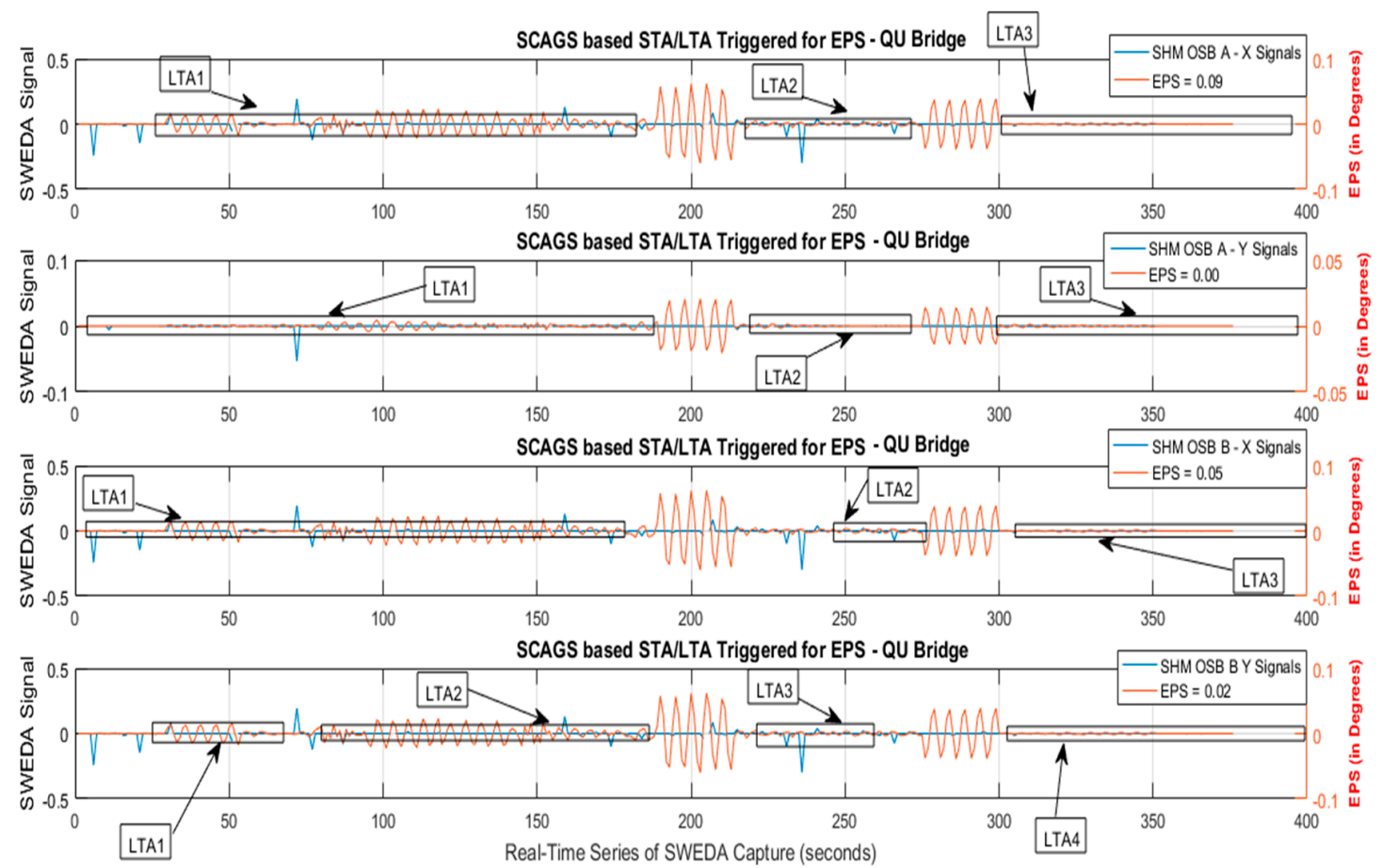

Figure 24. Earthquake probabilistic sequence (EPS) for the assumed dataset (down-sampled to 400). 
In Figure 25, the final output of SWEDA is displayed for the QU Bridge site. The EPS values $(0.01,0.00,0.00,0.00)$ show that for the current situation the percentage resemblance with seismic waves is 0.00 as there was not a single STA detected from the six waves stored SCAGS. The higher or longer the length of the LTA, the closer the signal to zero. At any point when red (EPS) intersects with blue (signal) and the part resembles even 73\%, it maps an STA. The absence of STAs shows neither P, nor $\mathrm{S}$, nor surface waves were detected. In fact, it may also reflect that the stored wave is an anomaly and may or may not be any seismic wave in the worst case.
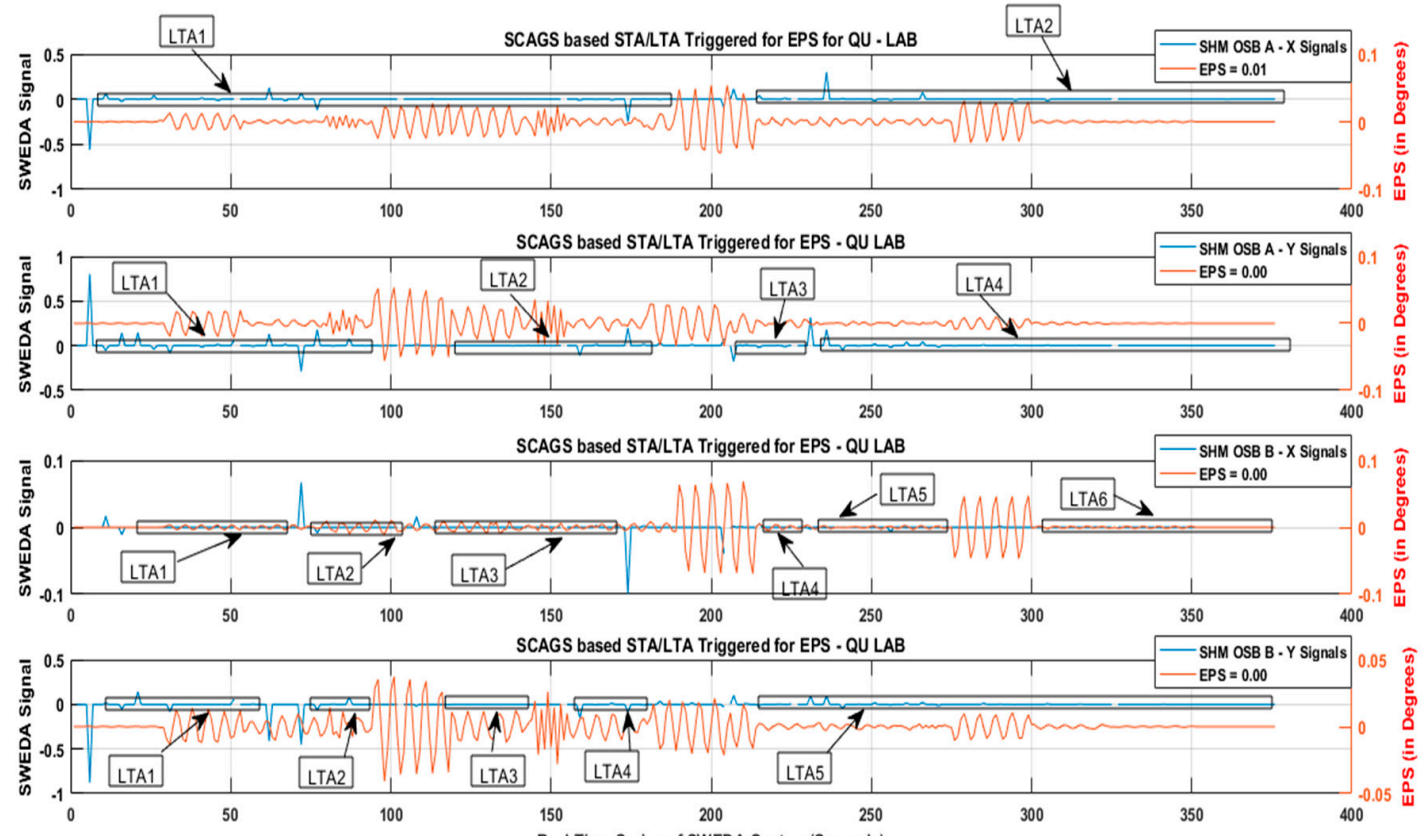

Figure 25. EPS for the assumed dataset (down-sampled to 400).

In Figures 14-25 SWEDA detected no signs of earthquake. In Figure 26, an induced earthquake was tested in-situ test bench for sensor nodes clusters set has been selected to test the performance of our SWEDA. Keeping an account of Equation (1), STA was triggered at $10 \mathrm{~s}$ and recorded eleven p-waves, second was triggered at $21 \mathrm{~s}$ and recorded $5 \mathrm{~s}$-waves, likewise third was triggered and stored 5 surface waves.

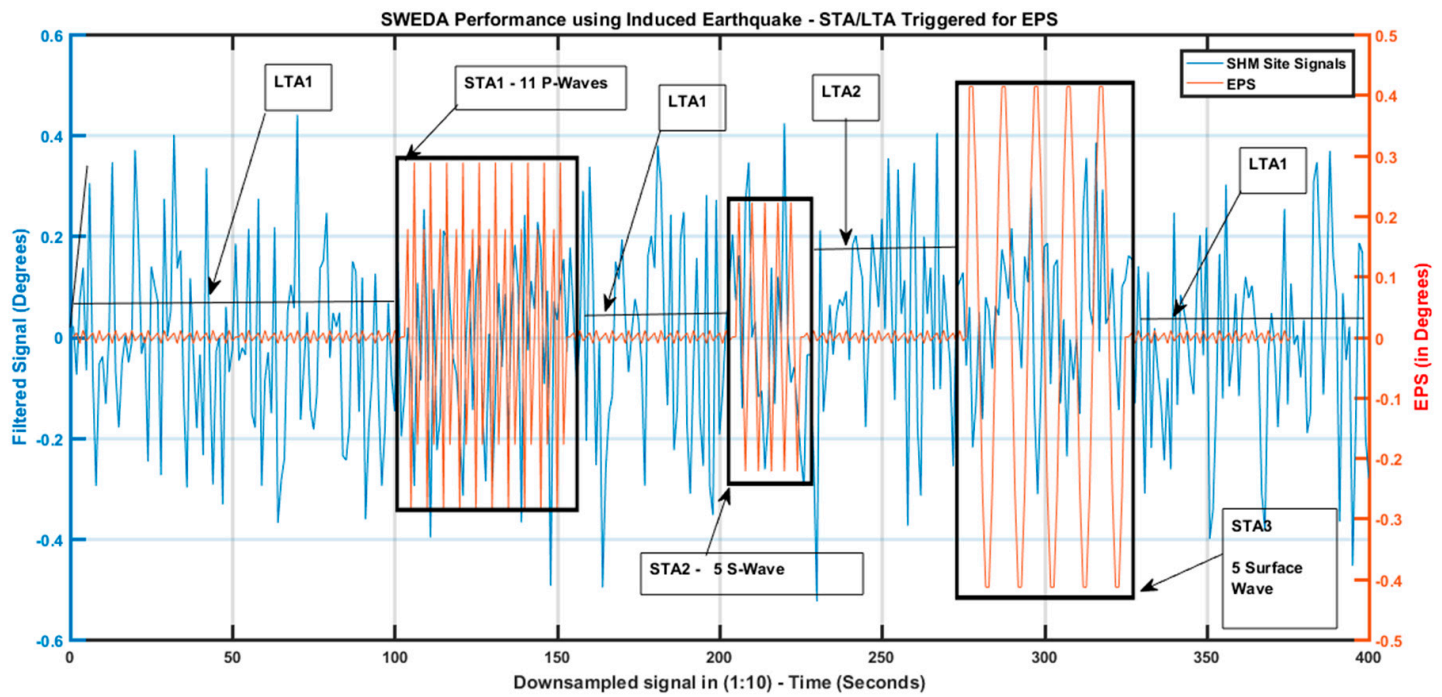

Figure 26. EPS for the assumed dataset (down-sampled to 400). 
PDS gave a scaling resolution command to ADCs from 16-bit to 22-bit to achieve that the CANopen baud rate of $250 \mathrm{~kb} / \mathrm{s}$ using $0 x 03$ was changed to $1 \mathrm{Mb} / \mathrm{s}$ using 0x00 SDO. The node, to be purely Industry 4.0 compliant, must maximize programmable and configurable hardware parameters. It is the minimum requirement to have programmable ADC and a soft configurable communication interface for context-aware situations. Every node is only transmitting the filtered word streams in binary format CANopen payload.

\section{Discussion and Limitations}

\subsection{The Problem/Challenge}

Regional events are happening in parallel at different geospatial locations. The tendency of similarity between these events is very high.

1. Which surface is suitable for seismic wave detection?

2. Which sensors are best for ground motion frequency, magnitude, and angle measurement?

3. How to align the crust or surface with sensors for ground motion detection?

4. How to reduce the computation by minimizing mathematical complexity?

5. How to detect if the disaster threshold is exceeded for the early warning?

6. How does the data need to be filtered for feature extraction and pattern recognition?

7. How to relate the curves that they can be decisive evidence of the seismic event?

8. What is the probability of an earthquake?

9. Which data structure is more suitable for the real-time accuracy of prediction?

10. What are the recommended specifications of the sensor resolution ideal for SWEDA?

11. What should be the communication configuration of urban-scale seismic sensor nodes for an efficient algorithmic response?

\subsection{The Contribution of Proposed SWEDA Application on SGBINs}

A campus-level SWEDA-SHM was designed. The inputs from two physically deployed SHM systems were used to test SWEDA, visible in Figure 14. The step-wise operations of SWEDA are given below:

1. Step 1: All the SGBIN data SP1 needed normalization so that the algorithm can be fed with maximum zeros to reduce the floating-point operations, as demonstrated in Figures 16 and 17.

2. Step 2: All the SGBIN SP1s outputs were filtered by SNF (Figure 21) using a MicroPython embedded filter signal. The savgol_filter eliminated unwanted frequencies by only allowing a range of 1 24 Hz. Furthermore, the ranges of more than $\pm 5^{\circ}$ amplitudes were eliminated, which were increasing the computation costs as well as directing towards false detection.

3. Step 3: All the SP2s were checked for unsafe thresholds, i.e., in the case of limestone in Qatar it was $\pm 0.57^{\circ}$. No emergency value was observed by SWEDA from SGBINs in SP1 and SP2 as exhibited in Figures 18-23.

4. Step 4: The unique peak detection operation generated SCAGS and triggering amplitudes for STA/LTA in SLAWS for a stationary signal, as no seismic conditions were observed by PDS in Figure 22.

5. Step 5: The runtime similarities estimation using ratio computation with SCAGS and SLAWS generated an EPS for an expected earthquake or seismic signal needed magnitude ratios as well as the signal clustering, presented in Figure 23 as the green gradient.

6. Step 6: The EPS was divided by SP2 to compute the maximum similarities for a possible earthquake for a computed EPS from stationary data. No earthquake was found in Figures 24 and 25 with EPS $=0.00-0.09$. 
The proposed SWEDA had two limitations that were observed during the implementation phase, which are given below:

1. The limitation of this work in the implementation phase is a dependency on proposed SGBINs.

2. The sequence of operations has to be observed in the same pattern.

\section{Future Recommendations}

This work can further be optimized for upcoming system-on-chip (SoC)-based smart sensing solutions with complex seismic detection applications in future works using S/L-Kurt [32] windows with the statistical pre-computation approach with an optimized sequence of algorithms mentioned in [37] and formulated comparisons.

\section{Conclusions}

This work proposes a real-time seismic wave event detection system using high precision inclinometers with novel enhancements in ubiquitous structural health monitoring systems, fostering the geo-seismic industry. The chained mechanism proposed, detected, and identified primary (or compression), secondary (or shear), and Love waves in time series sensor data of 500 samples and real-time sensor time series of 80,000 samples from the Local Laboratory and Bridge sites. The systematically ordered process was initiated with a gradient map auto-calibration that eradicated the heterogeneous sensor placement plane errors and centered the inclinometer's $x$-axis at $y=0$, followed by data refinement by impairing noise of frequency higher than $24 \mathrm{~Hz}$ using a de-noising filter. Further, a histogram for magnitude density or occurrence $(-0.47,-0.2,0.23,0.27$, and 0.02 degrees) appeared for 71,000 to 73,000 samples, and FFT was carried out for frequencies $<24 \mathrm{~Hz}$ as a dual verification benchmark. In addition, real-time pre-triggering and post-triggering magnitudes for recorder tools were initiated by peak detection sequences for given geological and geographical parameters of the seismic wave nature. The expected seismic probabilistic wave construction was constituted by an auto-regressive pattern mapping sequence for real-time similarity and a $0.71 \sim 1.2$ ratio for the earthquake computation. Moreover, further optimization was achieved using window definitions for the wave characterization using the scaling coefficients array generation sequence. Almost exact STA/LTA windows mapped over real-time signals, and the predicted seismic sequence was observed for the Local Laboratory case for up to five LTA triggers with a maximum earthquake probabilistic sequence similarity of 0.01 and for three LTA triggers for the Local Bridge case with a maximum EPS similarity of 0.09 . The main contribution of this work is a triangular synergy of geophysics, instrumentation, and data processing. This work is a futuristic staircase in real-time detection and the study of natural disasters using state-of-the-art applied sciences and methods for earth and planetary research and development.

Author Contributions: Conceptualization, H.T.; Data curation, H.T.; Formal analysis, H.T.; Funding acquisition, F.T., M.A.E.A.-H., D.C. and A.B.M.; Investigation, H.T; Methodology, H.T.; Project administration, F.T.; Resources, F.T., M.A.E.A.-H., D.C. and A.B.M.; Software, H.T.; Supervision, F.T.; Validation, H.T.; Visualization, H.T.; Writing—original draft, H.T.; Writing—review \& editing, F.T., M.A.E.A.-H., D.C. and A.B.M.

Funding: This publication was made possible by the NPRP grant \# 8-1781-2-725 from the Qatar National Research Fund (a member of Qatar Foundation). The statements made herein are solely the responsibility of the authors.

Conflicts of Interest: The authors declare no conflict of interest. The funders had no role in the design of the study; in the collection, analyses, or interpretation of data; in the writing of the manuscript, or in the decision to publish the results.

\section{References}

1. Herlander, M.; Andreilcy, A.; Adilson, P.; Abel, M.; José, A.A. Impacts of Natural Disasters on Environmental and Socio-Economic Systems: What makes the difference? Ambiente Soc. 2013, 16, 45-64.

2. Franco, A.; Peter, N.; Frits, J.S. The Challenge of Sustainable Development. In Economy and Ecology: Towards Sustainable Development. Economy \& Environment; Springer: Dordrecht, The Netherlands, 1989. 
3. Mario, A.R.E.; Donghyun, P. A New Assessment to Measure the Risk Levels between Natural Disasters and Socio- Economic-Political Disasters. Available SSRN 3400582 2019. [CrossRef]

4. Wenzhan, S.; Fangyu, L.; Maria, V.; Liang, Z. Toward Creating a Subsurface Camera. Sensors 2019, 19, 301.

5. Abdurrahman, S.; Rafet, S.; Aysegul, A.; Muneo, H. Development of Integrated Earthquake Simulation System for Istanbul. Earth Planets Space 2016, 68, 115.

6. Hasan, T.; Farid, T.; Damiano, C.; Adel, B.M. Design and Implementation of Programmable Multi-Parametric 4-Degrees of Freedom Seismic Waves Ground Motion Simulation IoT Platform. In Proceedings of the Fifteenth International Wireless Communications \& Mobile Computing Conference (IWCMC), Tangier, Morocco, 24-28 June 2019.

7. Öcal, N. Design and Challenges for a Tsunami Early Warning System in the Marmara Sea. Earth Planets Space 2016, 68, 13.

8. Christelle, S.; Pierre-Yves, B.; Bertrand, G.; Jacques, H.; Cécile, C.; Jocelyne, G.; Michelle, A. Using Ambient Vibration Measurements for Risk Assessment at an Urban Scale: From Numerical Proof of Concept to Beirut Case Study (Lebanon). Earth Planets Space 2017, 69, 60.

9. Hasan, T.; Anas, T.; Farid, T.; Mohammed, A.A.; Adel, B.M.; Damiano, C. Geographical Area Network-Structural Health Monitoring Utility Computing Model. Int. J. Geo Inf. 2018, 8, 154.

10. Ware, R.H.; Fulker, D.W.; Stein, S.A.; Anderson, D.N.; Avery, S.K.; Clark, R.D.; Droegemeier, K.K.; Kuettner, J.P.; Minster, J.; Sorooshian, S. Real-time National GPS Networks: Opportunities for Atmospheric Sensing. Earth Planets Space 2019, 52, 901-905. [CrossRef]

11. Arvid, H. Civil Structural Health Monitoring Strategies, Methods and Applications. Ph.D. Thesis, Luleå Tekniska Universitet, Luleå, Sweden, 2007.

12. Farid, T.; Hasan, T.; Mohammed, A.A.; Adel, B.M.; Damiano, C. Design and Simulation of a Green Bi-Variable Mono-Parametric SHM Node and Early Seismic Warning Algorithm for Wave Identification and Scattering. In Proceedings of the 14th International Wireless Communications \& Mobile Computing Conference (IWCMC), Limassol, Cyprus, 25-29 June 2018. [CrossRef]

13. Farid, T.; Hasan, T.; Mohammed, A.A.; Adel, B.M.; Anas, T.; Damiano, C. IoT and IoE prototype for scalable infrastructures, architectures and platforms. Int. Robot. Autom. J. 2018. [CrossRef]

14. Farid, T.; Hasan, T.; Adel, B.M.; Damiano, C. Development of Prototype for IoT and IoE Scalable Infrastructures, Architectures and Platforms. In Proceedings of the Fourth International Symposium, Ubiquitous Networking, Hammamet, Tunisia, 2-5 May 2018. [CrossRef]

15. Eric, N.H.; Mohammed, S.H. Gator: An Optimized Discrimination Network for Active Database Rule Condition Testing. Master's Thesis, CIS Department University of Florida, Gainesville, FL, USA, 1993.

16. Hasan, T.; Anas, T.; Farid, T.; Mohammed, A.A.; Adel, B.M.; Damiano, C. Structural Health Monitoring Installation and Deployment Scheme using Utility Computing Model. In Proceedings of the Second European Conference in EECS, Bern, Switzerland, 20-22 December 2018.

17. Mohammad, A.H.; Mohammad, K.H.; Pin, J.K.H. A review on sensors and systems in structural health monitoring: Current issues and challenges. Smart Struct. Syst. 2018, 22, 509-525.

18. Arvind, D.; Ali, D.; Chun, H.W.; Sabu, J. A Review of Passive Wireless Sensors for Structural Health Monitoring. Mod. Appl. Sci. 2013, 7, 57-76.

19. Maria, L.; Aldo, Z. A Bayesian Approach to the Real-time Estimation of Magnitude from the Early P and S Wave Displacement Peaks. J. Geophys. Res. Atmos. 2008. [CrossRef]

20. Wu, H.; Zhang, B.; Li, F.; Liu, N. Semi-automatic first arrival picking of micro-seismic events by using pixel-wise convolutional image segmentation. Geophysics 2019, 84, V143-V155. [CrossRef]

21. Zefeng, L.; Men-Andrin, M.; Egill, H.; Zhongwen, Z.; Jennifer, A. Machine Learning Seismic Wave Discrimination: Application to Earthquake Early Warning. Geophys. Res. Lett. 2018, 45, 4773-4779.

22. Ioannis, A.; Ioannis, T.; Anaxagoras, E. Intelligent Seismic Acceleration Signal Processing for Damage Classification in Buildings. IEEE Trans. Instrum. Meas. 2007, 56, 1555-1564.

23. Niksa, O.; Sven, L. Earthquake-explosion Discrimination using Genetic Algorithm based Boosting Approach. Comput. Geosci. 2010, 36, 179-185.

24. Conny, H.; Moritz, B.; Matthias, O. A seismic event spotting system for volcano fast response systems. Bull. Seismol. Soc. Am. 2012, 102, 948-960.

25. Silvia, S.F.; Eugène, C.E.; Mohammad, I.M. Automatic Classification of Seismic Signals at Mt. Vesuvius Volcano, Italy, using Neural Networks. Bull. Seismol. Soc. Am. 2005, 95, 185-196. 
26. Cheng, T.; Brian, L.N.K. Automatic Seismic Event Recognition and Later Phase Identification for Broadband Seismograms. Bull. Seismol. Soc. Am. 2009, 86, 1896-1909.

27. Claudio, S.; Yih-Min, W.; Aldo, Z.; Hiroo, K. Earthquake Early Warning: Concepts, Methods and Physical Grounds. Soil Dyn. Earthq. Eng. 2011, 31, 106-118.

28. Jiang, W.; Yu, H.; Li, L.; Huang, L. A Robust Algorithm for Earthquake Detector. In Proceedings of the World Congress on Engineering Education (WCEE), Beirut, Lebanon, 29-30 October 2015.

29. Mitchell, W.; Richard, A.; Christopher, Y.; Judy, B.; Mark, H.; Susan, M.; Julian, T. A Comparison of Select Trigger Algorithms for Automated Global Seismic Phase and Event Detection. Bull. Seismol. Soc. Am. 1998, 88, 95-106.

30. Satish, K.; Renu, V.; Pawan, K. Development of Earthquake Event Detection Technique Based on STA/LTA Algorithm for Seismic Alert System. Geo. Soc. India 2018, 92, 679-686.

31. Joshua, P.J.; Mirko, B. Adaptive STA-LTA with Outlier Statistics. Bull. Seismol. Soc. Am. 2015, 105, 1606-1618.

32. Fangyu, L. Automatic Arrival Identification System for Real-time Microseismic Event Location. In Proceedings of the SEG International Exposition and 87th Annual Meeting, Houston, TX, USA, 24-27 September 2017.

33. Fangyu, L.; Jamie, R.; Kurt, J.M.; Huailai, Z. Automatic event detection on noisy microseismograms. In Proceedings of the 84th Annual International Meeting, SEG, Expanded Abstracts, Las Vegas, NV, USA, 5 August 2014.

34. Anthony, L.; Claudio, S.; Maurizio, V. Automatic Picker Developments and Optimization: FilterPicker-A Robust, Broadband Picker for Real-time Seismic Monitoring and Earthquake Early-Warning. Seismol. Res. Lett. 2012, 83, 531-540.

35. Ruano, A.E.; Madureira, G.; Barros, O. Seismic Detection using Support Vector Machines. J. Neurocomputing 2013, 135, 273-283. [CrossRef]

36. Serdar, K.H.; Richard, M.A.; Holly, B.; Margaret, H.; Ivan, H.; Douglas, N. Designing a Network-Based Earthquake Early Warning Algorithm for California: ElarmS-2. Bull. Seismol. Soc. Am. 2013, 104, 162-173. [CrossRef]

37. Jubran, A.; David, W.E. A review and appraisal of arrival-time picking methods for downhole microseismic data. Geophysics 2016, 81, KS71-KS91.

38. Thomas, B.; Mike, E.D. Normalized Iterative Hard Thresholding: Guaranteed Stability and Performance. Ieee J. Sel. Top. Signal. Process. 2009, 4, 298-309.

39. Jonathan, B.; Jonathan, M.B. Two-point step size gradient methods. IMA J. Numer. Anal. 1998, 8, 141-148.

40. Sleeman, T.; Torild, V.E. Robust automatic P-phase picking: An on-line implementation in the analysis of broadband seismogram recordings. Phys. Earth Planet. Int. 1999, 113, 265-275. [CrossRef]

41. Dias, P.J.M.; Postolache, O.; Girão, P.M. Digitally Programmable A/D Converter for Smart Sensors Applications. IEEE Trans. Instrum. Meas. 2017, 56, 158-163. [CrossRef]

42. Peter, J.; Cees, V.L. OMPC: An Open-Source MATLAB-to-Python Compiler. Front. Neuroinformatics $2007,3,5$.

43. Masatoshi, M. Detection of seismic events triggered by P-waves from the 2011 Tohoku-Oki earthquake. Earth Planets Space 2013, 64, 16.

44. Lucia, M.; Leif, W.; John, B. A Comparison of Coda and S-Wave Spectral Ratios as Estimates of Site Response in the Southern San Francisco Bay Area. Bull. Seismol. Soc. Am. 1994, 84, 1815-1830.

45. Fan-Chi, L.; Morgan, P.M.; Michael, H.R. Surface wave Tomography of the Western United States from Ambient Seismic Noise: Rayleigh and Love wave Phase Velocity Maps. Geophys. J. Int. 2007, 173, 281-298.

(C) 2019 by the authors. Licensee MDPI, Basel, Switzerland. This article is an open access article distributed under the terms and conditions of the Creative Commons Attribution (CC BY) license (http://creativecommons.org/licenses/by/4.0/). 